\title{
Human Effector Memory T Helper Cells Engage with Mouse Macrophages and Cause Graft-versus- Host-Like Pathology in Skin of Humanized Mice Used in a Nonclinical Immunization Study
}

Balasai Sundarasetty, ${ }^{* \dagger}$ Valery Volk, ${ }^{* \dagger}$ Sebastian J. Theobald, ${ }^{* \dagger}$ Susanne Rittinghausen, ${ }^{\ddagger}$ Dirk Schaudien, ${ }^{\ddagger}$ Vanessa Neuhaus, ${ }^{\S}$ Constanca Figueiredo, ${ }^{\oplus}$ Andreas Schneider, ${ }^{\dagger \dagger}$ Laura Gerasch, ${ }^{* \dagger}$ Adele Mucci, ${ }^{\|}$Thomas Moritz, $\|$Constantin von Kaisenberg, ${ }^{* *}$ Loukia M. Spineli, ${ }^{\dagger \dagger}$ Katherina Sewald, ${ }^{\S}$ Armin Braun, ${ }^{\S}$ Henning Weigt, ${ }^{\S}$ Arnold Ganser, ${ }^{\dagger}$ and Renata Stripecke ${ }^{* \dagger}$

From Regenerative Biology to Reconstructive Therapies $($ REBIRTH),* Laboratory of Regenerative Immune Therapies Applied, the Departments of
Hematology, Hemostasis, Oncology and Stem Cell Transplantation ${ }^{\dagger}$ and Transfusion Medicine, ${ }^{\uparrow}$ the Institutes of Experimental Hematology ${ }^{\|}$and
Biostatistics, ${ }^{\dagger \dagger}$ and the Clinic of Gynecology and Obstetrics, ${ }^{* *}$ Hannover Medical School, Hannover; and the Departments of Pathology $y^{\ddagger}$ and Airway
Immunology, ${ }^{\S}$ Fraunhofer Institute for Toxicology and Experimental Medicine Hannover, Hannover, Germany

Accepted for publication

February 16, 2017.

Address correspondence to Renata Stripecke, Ph.D., Regenerative Biology to Reconstructive Therapies (REBIRTH), Laboratory of Regenerative Immune Therapies Applied, Department of Hematology, Hemostasis, Oncology and Stem Cell Transplantation, Hannover Medical School, OE6862, Hans Borst Zentrum, Carl Neuberg Strasse 1, D-30625 Hannover, Germany.

E-mail: stripecke.renata@mhhannover.de.

\begin{abstract}
Humanized mice engrafted with human hematopoietic stem cells and developing functional human T-cell adaptive responses are in critical demand to test human-specific therapeutics. We previously showed that humanized mice immunized with long-lived induced-dendritic cells loaded with the pp65 viral antigen (iDCpp65) exhibited a faster development and maturation of T cells. Herein, we evaluated these effects in a long-term (36 weeks) nonclinical model using two stem cell donors to assess efficacy and safety. Relative to baseline, iDCpp65 immunization boosted the output of effector memory $\mathrm{CD}_{4}{ }^{+} \mathrm{T}$ cells in peripheral blood and lymph nodes. No weight loss, human malignancies, or systemic graft-versus-host (GVH) disease were observed. However, for one reconstitution cohort, some mice immunized with iDCpp65 showed GVH-like signs on the skin. Histopathology analyses of the inflamed skin revealed intrafollicular and perifollicular human $\mathrm{CD4}^{+}$cells near $\mathrm{F} 4 / 80^{+}$mouse macrophages around hair follicles. In spleen, $\mathrm{CD}^{+}$cells formed large clusters surrounded by mouse macrophages. In plasma, high levels of human $\mathrm{T}$ helper 2-type inflammatory cytokines were detectable, which activated in vitro the STAT5 pathway of murine macrophages. Despite this inflammatory pattern, human $\mathrm{CD}^{+} \mathrm{T}$ cells from mice with GVH reacted against the pp65 antigen in vitro. These results uncover a dynamic cross-species interaction between human memory $\mathrm{T}$ cells and mouse macrophages in the skin and lymphatic tissues of humanized mice. (Am J Pathol 2017, 187: 1380-1398; http://dx.doi.org/10.1016/j.ajpath.2017.02.015)
\end{abstract}

Novel biomedical products for human use, such as monoclonal antibodies, engineered $\mathrm{T}$ cells expressing chimeric antigen receptors, and novel vaccines, are overdue in their translation to clinics, partly because of the limitations of appropriate human-specific preclinical models to assess their immunological efficacy and safety. Small animal models composed of immune-deficient mouse models reconstituted with human hematopoietic stem cells (Hu-HSCs) are therefore excellent options for such studies. Hu-HSC mice can potentially replace costly and demanding studies that use nonhuman primates to characterize the
Supported by the German Research Council Regenerative Biology to Reconstructive Therapies (REBIRTH) Cluster of Excellence, Units 6.4. (R.S.), 6.3., (C.F.), 1.3 (T.M.), 9.4 (K.S. and A.B.); German Center for Infections Research (DZIF) Hannover Region grant TTU07.803 (R.S.); German Research Council Collaborative Research Center 738 Project A6 (R.S.) and Project C4 (T.M.). V.V. received a fellowship from the Center of Infections Biology Ph.D. Program (ZIB) and S.J.T. received a fellowship from the Regenerative Sciences Ph.D. Program (RegSci).

B.S. and V.V. contributed equally to this work.

Disclosures: A.G. and R.S. are currently applying for a patent related to the content of the article, titled "Induced dendritic cells and uses thereof." R.S. received honoraria from The Jackson Laboratory for lectures.

Current address of B.S., Institute for Molecular Medicine, University Hospital Frankfurt, Frankfurt am Main, Germany. 
spatiotemporal immunological events in vivo. ${ }^{1,2}$ Several highly immunodeficient mouse strains have been developed and improved in the past decade, such as the nonobese diabetic (NOD)/severe combined immunodeficiency (SCID)/ $\mathrm{IL}-2 \mathrm{R} \gamma \mathrm{c}^{\text {null }}$ and NOD/Rag $1^{\text {null }} / \mathrm{IL}-2 \mathrm{R} \gamma \mathrm{c}^{\text {null }}$ (NRG) mice, which completely lack lymphocytes (T, B, and natural killer cells) and therefore support robust engraftment of Hu-HSCs. An important reason for the success of Hu-HSC engraftment in the NOD-derived mutant strains is the signal regulatory protein $\alpha$-CD47 receptor-ligand interaction, which provides the do not eat me signal to mouse macrophages, avoiding the rejection of the human hematopoietic system by murine phagocytosis. ${ }^{3}$ Development of human myeloid and lymphoid cells in the murine lymphatic tissues and subsequent homing in several organ systems has been extensively characterized in Hu-HSC mice. ${ }^{2}$ However, the interplay between the human lymphocytes and the murine myeloid cells, particularly because they are completely mismatched for the major histocompatibility complex, is still not clearly understood. Most studies using these models are performed for approximately 15 to 20 weeks after HSC transplantation (HSCT). At this time, the human T-cell maturation observed in the lymphatic tissues is still incomplete, probably because of the low levels of mature human professional antigen-presenting cells (APCs), such as dendritic cells (DCs) in murine lymphatic tissues. We have previously shown significantly accelerated development of the human adaptive immune responses in $\mathrm{Hu}-$ HSC mice transplanted with peripheral blood or cord blood- derived $\mathrm{CD} 34^{+}$cells that were then administered with autologously engineered induced DCs (iDCs). ${ }^{4,5}$ These iDCs consisted of monocytes that were transduced with lentiviral vectors and coexpressed granulocyte-macrophage colonystimulating factor (GM-CSF) and interferon $\alpha$ (IFN- $\alpha$ ), and were endogenously loaded with the immune dominant antigen pp65 from the human cytomegalovirus. In vitro, the transduced monocytes showed autonomous DC differentiation, maturation, and activation of T cells. ${ }^{6}$ Thus, iDCs tested in Hu-HSC mice served as a proof-of-concept model for a cellular vaccine currently being developed in our laboratory for translation into immunization of immunocompromised patients at high risk of human cytomegalovirus reactivation after HSCT. $^{7}$ We demonstrated remarkable effects once iDCs self-differentiated in vivo, maintaining high viability for up to 2 weeks. iDCs migrated effectively to the lymph node Anlage, mediating T- and B-cell activation, expansion, and antigen-specific immune responses. iDCs also augmented the thymic and extrathymic development of naïve $\mathrm{T}$ cells. These results supported the hypothesis that the human lymphocytes required supplementation with human iDCs for their improved major histocompatibility complexdependent maturation in lymphopenic Hu-HSC mouse models. We thus obtained scientific advice from the German office of the European Medicines Agency regarding further nonclinical in vivo testing of iDCs for clinical development. The recommendation was to evaluate potential occurrence of immune pathologies or malignancies in a long-term model [ie, to extend the experimental observation time of the Hu-HSC model from 10 to 26 weeks (ie, 6 months) after the first iDC immunization]. In addition, studies should be performed under good laboratory practices. Therefore, in collaboration with a contract research organization, we run pilot feasibility studies of long-term analyses of human immune reconstitution after iDC administration. NRG mice were transplanted with highly pure $\mathrm{CD} 34^{+}$cells from two cord blood (CB) donors and showed robust engraftment of human $\mathrm{CD} 45^{+}$ cells. A cohort of mice was prime boosted with cryopreserved/thawed induced dendritic cells expressing pp65 (iDCpp65) and kept under observation for 26 weeks. Longitudinal analyses of the blood samples were performed to follow the development and maturation of human $\mathrm{T}$ cells. The mice were continuously monitored for any signs of pathologies. At the end point of the studies, as expected, we documented the infiltration and maturation of human $\mathrm{T}$ cells in the lymphatic tissues, but no systemic graft-versus-host $(\mathrm{GVH})$ disease. We report herein that three of nine mice immunized with iDCpp65 showed GVH-like signs on the skin (GVH-S). The pathology was associated with high infiltration of human $\mathrm{CD} 4^{+}$cells in tissues and detection of human T helper-2 (Th2) type cytokines in plasma. Concurrently, accumulation of mouse macrophages was observed in affected areas of skin and surrounding human $\mathrm{CD} 4^{+} \mathrm{T}$ cells in the spleen. These observations supported the hypothesis that human cytokines can activate mouse macrophages in humanized mice, which we then confirmed with an in vitro assay. Therefore, our results serve as a note of caution when using long-term Hu-HSC mice as nonclinical models to predict pathologies that can be caused by adoptive human immune cells. Even so, the efficacy of iDCpp65 was further extended, as human pp65-reactive long-term memory $\mathrm{T}$ cells could be detected even 6 months after the first iDCpp65 immunization.

\section{Materials and Methods}

\section{Cell Lines}

The HEK-293 (human embryonic kidney-293) cell line encoding the simian virus 40 (SV40) large $\mathrm{T}$ antigen (heretofore, 293T cells) was used for the production of lentiviral vectors. $293 \mathrm{~T}$ cells were cultured at $37^{\circ} \mathrm{C}$ and $5 \%$ $\mathrm{CO}_{2}$ in Dulbecco's modified Eagle's medium (Life Technologies/Thermo Fisher Sceintific, Darmstadt, Germany) supplemented with 10\% fetal bovine serum (HyClone/GE Healthcare Life Sciences, Logan, UT). K562 cells expressing HLA-A*02.01 (KA2) and KA2 coexpressing CMV phosphoprotein 65 (pp65) expression were described ${ }^{4,5}$ and cultured in RPMI 1640 medium supplemented with 10\% fetal bovine serum, $1 \%$ penicillin/streptomycin, and $1 \mathrm{mg} /$ $\mathrm{mL}$ geneticin (Life Technologies $\mathrm{GmbH}$ ). RAW264.7 (mouse macrophage cell line) was cultured in Dulbecco's modified Eagle's medium supplemented with $10 \%$ fetal bovine serum and $1 \%$ penicillin/streptomycin. 


\section{Production and Titration of IDLV}

A third-generation self-inactivating integrase-defective lentiviral vector (IDLV) coexpressing GM-CSF, IFN- $\alpha$, and pp65 was generated and titrated by quantifying the p 24 HIV-I core protein by ELISA, as previously described. ${ }^{4,7}$

\section{iDCpp65 Generation and Administration}

Umbilical CB units were obtained under informed consent of the mothers in accordance with study protocols approved by Hannover Medical School Ethics Review Board. Mononuclear cells were isolated by Ficoll gradient centrifugation, $\mathrm{CD} 34^{+}$cells were selected after two rounds of immune-magnetic bead isolation (Miltenyi Biotech, Bergisch-Gladbach, Germany), and both $\mathrm{CD}_{3} 4^{+}$and CD $34^{-}$fractions were cryopreserved. A bank of CD $34^{+}$ cells established in our laboratory was used for pretesting and selection of the $\mathrm{CB}$ units that resulted into reproducible engraftment and persistency of human $\mathrm{CD} 45^{+}$cells $(>20 \%$ huCD $\left.45^{+}\right)$and reconstitution of lymphocytes $\left(\mathrm{CD}^{+}\right.$, $\mathrm{CD} 4^{+}, \mathrm{CD}^{+}, \mathrm{CD} 19^{+}$) in HIS mice for at least 15 weeks after HSCT. Two units positive for HLA-A*02.01 and showing persistency of huCD45 ${ }^{+}$in peripheral blood lymphocytes (PBL) and tissues for at least 15 weeks were chosen. $\mathrm{CD} 14^{+}$monocytes were isolated from the $\mathrm{CD} 34^{-}$ fractions for the production of iDCpp65, as described. ${ }^{4,5}$ Briefly, CD14 ${ }^{+}$cells were isolated using CD14 isolation beads (Miltenyi Biotech). The monocytes were preconditioned with recombinant human GM-CSF and IL-4 (50 ng/ $\mathrm{mL}$ each; Cellgenix, Freiburg, Germany) in X-VIVO 15 medium (Lonza GmbH, Cologne, Germany) for 8 hours before transduction in an incubator at $37^{\circ} \mathrm{C}$ and $5 \% \mathrm{CO}_{2}$. The $2.5 \mu \mathrm{g} / \mathrm{mL}$ p24 equivalent of the ID-LV-G2 $\alpha-\mathrm{pp} 65$ vector was used to transduce $5 \times 10^{6}$ monocytes in the presence of $5 \mu \mathrm{g} / \mathrm{mL}$ protamine sulfate (Valeant, Eschborn, Germany) for 16 hours. After transduction, monocytes were harvested, resuspended in freezing medium containing $15.5 \%$ human albumin, $10 \%$ dimethyl sulfoxide, and 5\% glucose, and cryopreserved at $-152^{\circ} \mathrm{C}$. Before immunization, the transduced cells (iDCpp65) were thawed, resuspended in phosphate-buffered saline (PBS), washed twice with PBS, and the viable cell counts were determined by trypan blue exclusion. A total of $5 \times 10^{5}$ viable cells were injected s.c. near the inguinal region as bilateral prime and boost administrations performed at 10 and 11 weeks after HSCT (described further below).

\section{Quality Control of Thawed iDCpp65}

Cryopreserved iDCpp65 was thawed and cultured for 7 days in X-VIVO 15 medium (Lonza) without the exogenous addition of cytokines. Surface marker expression was analyzed by flow cytometry using the monoclonal antibodies conjugated with fluorochromes: fluorescein isothiocyanate-conjugated antiCD14 (Miltenyi Biotec), phosphatidylethanolamine- conjugated anti-CD80, PerCP-conjugated anti-HLA-DR, and APC-conjugated anti-CD86 (BD Biosciences, Heidelberg, Germany). Nontransduced monocytes and monocytes cultured in X-VIVO 15 with exogenous addition of GM-CSF and IFN$\alpha$ (conventional IFN- $\alpha$ DCs) were used as negative and positive controls, respectively. Acquisitions and analyses were performed using an LSRII Flow Cytometer (BD Biosciences) and FlowJo analysis software version 7.6.4 (Tree Star Inc., Ashland, OR). The IDLV copy numbers in transduced monocytes were quantified by real-time quantitative PCR, as previously described. ${ }^{7}$ Samples and data were analyzed with StepOnePlus Real-Time PCR system (Applied Biosystems, Life Technologies, Darmstadt, Germany).

\section{HSCT into NRG Mice}

All procedures involving mice were reviewed and approved by the Lower Saxony and followed the guidelines provided by the Animal Facility at the Hannover Medical School.

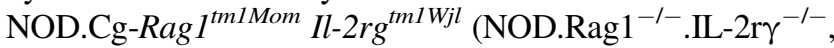
NRG) mice were obtained from The Jackson Laboratory (Bar Harbor, $\mathrm{MN}$ ) and bred and maintained under pathogenfree conditions in an IVC system (BioZone, Margate, UK) at Hannover Medical School. HSCT was performed as previously described. ${ }^{4,5}$ Shortly, $0.2 \times 10^{6} \mathrm{CD} 34^{+}$stem cells were injected through the tail vein into 4-week-old irradiated NRG mice (450 cGy; Gammacell 3000; Best Theratonics, Ottawa, Canada).

\section{Analysis of Immune Reconstitution by Flow Cytometry}

Peripheral blood was collected from surviving mice at weeks $10,15,20,25,30,35$, and 36 , and engraftment of human lymphocytes was analyzed by flow cytometry. At signs of debilitating pathology or at the end point analyses (36 weeks after HSCT), mice were sacrificed for collection of tissues. For flow cytometry analyses, blood, plasma, thymus, spleen, peripheral lymph nodes, mesenteric lymph nodes, and bone marrow specimens were processed as single-cell suspensions and the following antibodies were used: PB-conjugated anti-CD45, APC-conjugated anti-CD3, Alexa700-conjugated anti-CD19, APCCy7-conjugated anti-CD4, PCy7-conjugated anti-CD8, fluorescein isothiocyanate-conjugated anti-CD45RA, and PCy5conjugated anti-CD62L. T-cell subpopulations were defined as naïve $(\mathrm{N})$ for $\mathrm{CD}^{4} 5 \mathrm{RA}^{+} \mathrm{CD}^{-} 2 \mathrm{~L}^{+}$, as effector memory (EM) for CD45RA ${ }^{-} \mathrm{CD}^{-} \mathrm{L}^{-}$and central memory (CM) for $\mathrm{CD}^{2} 5 \mathrm{RA}^{-} \mathrm{CD} 2 \mathrm{~L}^{+}$cells. Analyses were performed in LSRII flow cytometer (BD Biosciences) using FlowJo software.

\section{Pathology, Histology, and Immunohistochemical Analysis}

We performed a complete necropsy on all euthanized animals. Organs and pathological lesions were fixed in $3.7 \%$ 
formaldehyde buffer (Carl Roth GmbH + Co KG, Karlsruhe, Germany) for 24 hours. The tissue material was then transferred to $70 \%$ ethanol for further fixation up to 48 hours. All macroscopic lesions, predefined parts of liver, spleen, kidney, colon, lung, brain, skin, and eyes with eyelids, were embedded in paraffin. Sections ( $3 \mu \mathrm{m}$ thick) from all organs were prepared and routinely stained with hematoxylin and eosin. Additional skin sections were stained with periodic acidSchiff, and liver sections were stained with Prussian blue. For histopathological evaluation of the skin, the grading of the occurrence of apoptotic cells was set as follows: very slight, up to two cells per high-power field (HPF); slight, up to five cells per HPF. The grading for the inflammatory reaction was very slight, with up to 10 inflammatory cells per HPF; slight, with up to 50 inflammatory cells per HPF; and moderate, with $>50$ inflammatory cells per HPF. For immunohistochemical detection of the chosen markers, paraffin slides ( $3 \mu \mathrm{m}$ thick) were sectioned from the histology wax blocks with the tissue material and mounted onto glass slides. Paraffin slides were then dewaxed and subjected to antigen retrieval methods, which had been validated for each of the primary antibodies for mouse and human tissues. Heat-induced antigen retrieval was performed in a citrate-buffered solution. All slides were rinsed with Tris-buffered saline (pH 7.6) plus $0.01 \%$ Tween 20 (Merck KGaA, Darmstadt, Germany; 8.22184). Slides were incubated for 20 minutes at $21^{\circ} \mathrm{C}$ in normal goat serum (Vector Laboratories Inc., Burlingame, CA; S-1000) and then incubated with the primary antibody for 1 hour at $21^{\circ} \mathrm{C}$ or 24 hours at $4^{\circ} \mathrm{C}$, respectively. As secondary antibodies, a biotin-SP-conjugated AffiniPure goat anti-mouse IgG (111-065-100), a goat anti-rabbit IgG (111-065-144), or a goat anti-rat IgG (705-066-147), which were all purchased from Jackson Immunoresearch (West Grove, PA), were applied for a 30-minute incubation time at $21^{\circ} \mathrm{C}$. Immunostaining was performed with a routine method using alkaline phosphatase streptavidin-biotin (Vector Laboratories Inc.; S-5100) and Fast Red as chromogen (Fast Red substrate pack; BioGenex, Freemont, CA; HK182-5K). The primary antibodies used for immunohistochemical analyses are listed in Table 1. The slides were finally counterstained with Bluing Reagent (Thermo Scientific, Braunschweig, Germany; 7301). Coverslipping was performed using Aquatex aqueous mounting medium (Merck KGaA, Darmstadt, Germany; 1.08562). Sample permeabilization, antibody concentrations, antibody reactions, and staining procedures were optimized for each antibody to get clear and specific immunohistochemical signals. We used a bright-field microscope for evaluation of the slides.

\section{Analysis of Human and Mouse Cytokines in Plasma}

Plasma was separated from peripheral blood and level of human Th1/Th2 cytokines was performed by fluorescent bead-based 14-plex Luminex assay, according to the manufacturer's instructions. The assay measured the following cytokines: GM-CSF, IL-4, tumor necrosis factor$\alpha$, IL-6, IL-8, monocyte chemoattractant protein-1 (MCP-1), IL-10, IL-1b, IL-5, IFN- $\gamma$, IL-7, IL-2, and IL-12p70 (Merck Millipore, Billerica, MA). Similarly, mouse cytokines in the plasma were analyzed for the following cytokines: GM-CSF, tumor necrosis factor- $\alpha$, IL-6, IL-8, IL-10, IL-5, IFN- $\gamma$, and IL-12p70, analyzed by multiplex assay (U-Plex; Meso Scale Discovery, Rockville, MD) and single plex MSD assay for MCP-1.

\section{Effect of Human Cytokines on RAW246.7 Cell Line}

RAW246.7 cells were seeded in a 6 -well plate $\left(1 \times 10^{6}\right.$ cells/well per $1 \mathrm{~mL}$ ), and recombinant human MCP-1, IL-6, IL-8, and IL-10 (50 ng/mL each; all cytokines are

Table 1 Antibodies Used for Immunohistochemical Analysis

\begin{tabular}{|c|c|c|c|}
\hline Antibody & Clone number & Manufacturer & Catalog number \\
\hline Anti-CD1a & 010 & Dako (Glostrup, Denmark) & M3571 \\
\hline Anti-CD20 & EP459Y & Abcam (Cambridge, UK) & ab78237 \\
\hline Anti-CD79-a & HM57 & Dako & M705 \\
\hline Anti-human CD11c & $5 \mathrm{D} 11$ & Cell Marque (Rocklin, CA) & $111 M-18$ \\
\hline Anti-human CD4 & EPR6855 & Abcam (Cambridge, UK) & ab133616 \\
\hline Anti-human glycophorin A & Polyclonal & Abcam & ab129024 \\
\hline Anti-human HLA-DR & EP96 & Epitomics (Burlingame, CA) & AC-0088RU0 \\
\hline Anti-human nuclear antigen antibody & $235-1$ & Abcam & ab191181 \\
\hline Anti-langerin & EPR15863 & Abcam & ab192027 \\
\hline Anti-mast cell tryptase & 10D11 & $\begin{array}{l}\text { Novocastra, Leica Microsystems } \\
\text { (UK) Ltd (Milton Keynes, UK) }\end{array}$ & NCL-MCTRYP \\
\hline Anti-S100 & Polyclonal & Dako & Z0311 \\
\hline
\end{tabular}


from PeproTech GmbH, Hamburg, Germany) were added to the wells in various combinations. Three hours after the cytokine treatment, cells were harvested and phoshpoSTAT5 (pSTAT5) up-regulation was analyzed by intracellular staining. Cells were washed once with PBS
(Biochrom GmbH, Berlin, Germany) and the cell pellet was resuspended in $100 \mu \mathrm{L} 4 \%$ paraformaldehyde (Carl Roth $\mathrm{GmbH}+\mathrm{Co} \mathrm{KG}$ ) and incubated at room temperature for 30 minutes. Cells were then washed with MACS buffer (Miltenyi Biotec) and the pellet was

A

HSCT

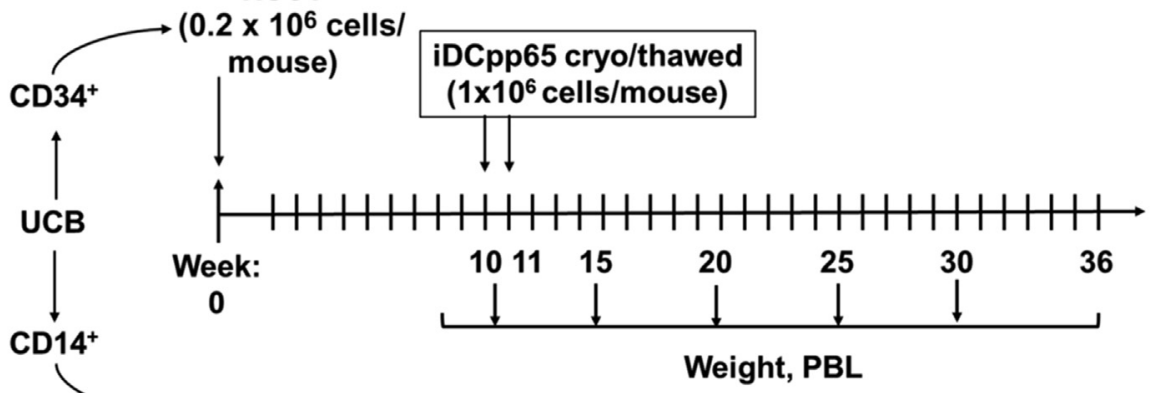

iDCpp65

B
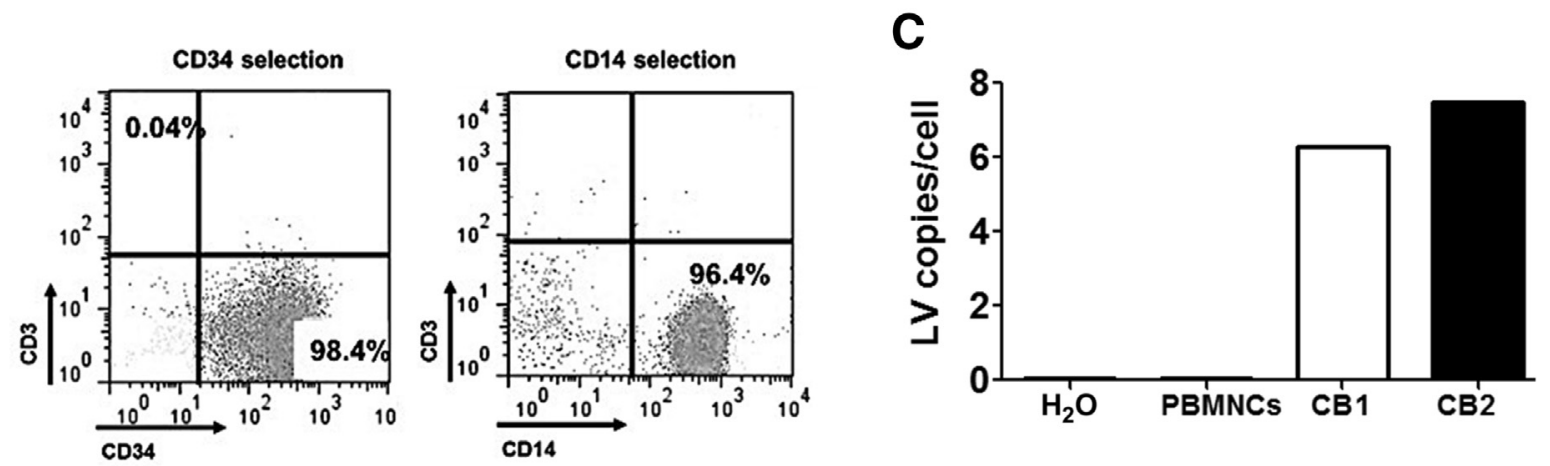

D

CD14

CD80

HLA-DR

CD86

CB 1
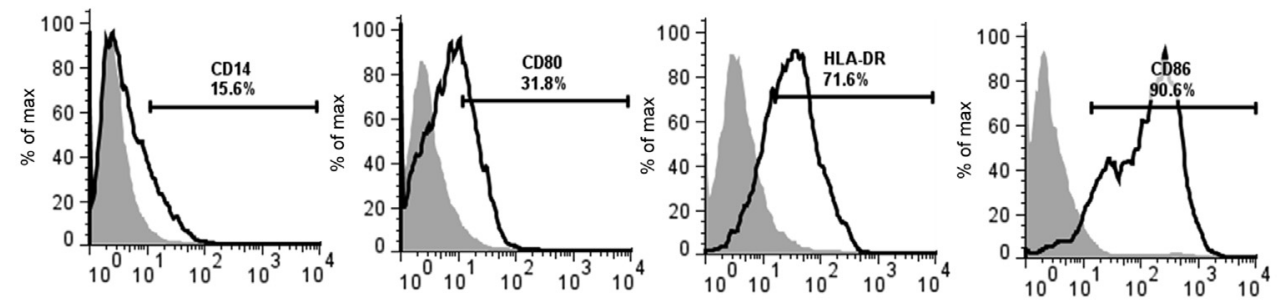

CB 2
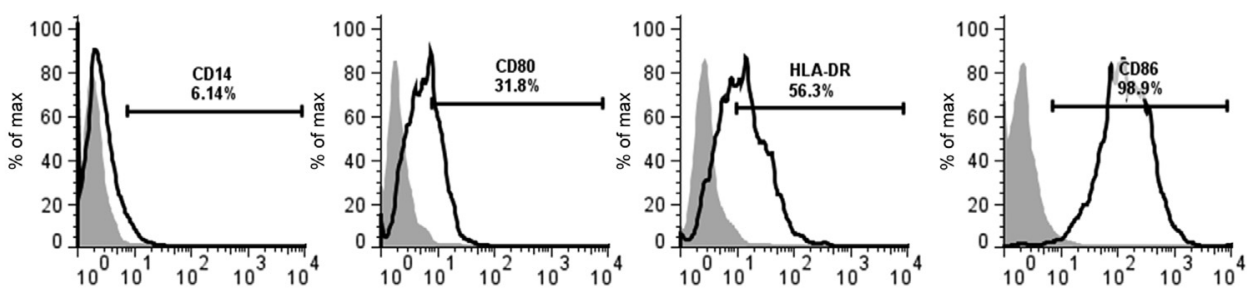

Figure 1 Nonclinical long-term study using NOD.Rag ${ }^{-/-} \mathrm{IL}-2 \gamma \mathrm{Cc}^{-/-}$mice transplanted with $\mathrm{CD}_{3} 4^{+}$stem cells from umbilical cord blood and immunized with iDCpp65. A: Scheme of transplantation, immunization, longitudinal, and end point analyses. Prime-boost immunization with iDCpp65 was performed at 10 and 11 weeks after transplantation, weight and peripheral blood lymphocytes (PBL) were monitored at weekly intervals, and weight, immune reconstitution in blood and tissues, histopathology, cytokines in plasma, and immunity against pp65 were assessed at end point analyses. B: Purity of CD34 ${ }^{+}-$and CD14 $^{+}-$ selected cells. Dot plots show the purity of ${\mathrm{CD} 34^{+}}^{+}$used for transplantation and $\mathrm{CD} 14^{+}$cells used for generation of iDCpp65. Flow cytometry analyses showed minimal contamination with $\mathrm{CD}^{+}$T cells. C: LV copies per cell on day 7 of culture. LV copies per cell were detected by quantitative RT-PCR in iDCpp65 generated with monocytes from cord blood (CB)1 and CB2 and maintained in culture for 7 days before analyses. D: Immunophenotype of iDCpp65 on day 7 of in vitro culture. Histograms show the immunophenotype of $\mathrm{DDCpp} 65$ generated with monocytes from CB1 and CB2 and maintained in culture for 7 days before flow cytometry analyses. Solid lines: CD14 is down-regulated, whereas the DC markers HLA-DR, CD86, and CD80 are highly expressed. Gray histograms indicate the staining with isotype control-conjugated antibodies. HSCT, hematopoietic stem cells transplantation; IHC, immunohistochemistry; LV, lentiviral vector; PBMNC, peripheral blood mononuclear cells; PBL, peripheral blood lymphocytes; QC, quality control. 
Table 2 Summary of the Skin Pathology Analysis with Scores and with the Main Immunohistological Findings Performed in the Skin and Spleen Sections of NRG Mice Transplanted with CB1

\begin{tabular}{|c|c|c|c|c|c|c|c|c|c|c|c|}
\hline $\begin{array}{l}\text { Group/week } \\
\text { sacrificed }\end{array}$ & $\begin{array}{l}\text { Mouse } \\
\text { ID/sex }\end{array}$ & $\begin{array}{l}\text { Erythema } \\
\text { snout/body* }\end{array}$ & $\begin{array}{l}\text { Skin } \\
\text { Inflammation }^{\dagger}\end{array}$ & $\begin{array}{l}\text { Skin } \\
\text { Apoptosis }\end{array}$ & Skin $M P O^{\S}$ & $\begin{array}{l}\text { Skin mouse } \\
\mathrm{F} 4 / 80^{\top}\end{array}$ & $\begin{array}{l}\text { Skin } \\
\text { human } \\
\text { CD68 }\end{array}$ & $\begin{array}{l}\text { Skin } \\
\text { human } \\
\text { CD11c }\end{array}$ & $\begin{array}{l}\text { Skin } \\
\text { human } \\
\text { CD4 }\end{array}$ & $\begin{array}{l}\text { Spleen } \\
\mathrm{MPO}^{\S}\end{array}$ & $\begin{array}{l}\text { Spleen } \\
\text { CD4 }\end{array}$ \\
\hline Control/36 & $1081 / M$ & $+/-$ & 3 & 2 & ++ & ++++ & + & + & ++++ & ++ & ++ \\
\hline iDCpp65/36 & $1075 / \mathrm{F}$ & $-1-$ & 1 & - & + & +++ & + & + & ++++ & ++ & ++++ \\
\hline iDCpp65/36 & $1076 / F$ & $+/-$ & 2 & - & ++ & ++++ & ++ & + & ++++ & ++ & ++++ \\
\hline iDCpp65/36 & $1078 / \mathrm{F}$ & $+/-$ & 2 & 2 & $++/+++$ & ++++ & + & $+/++$ & ++++ & ++ & ++ \\
\hline iDCpp65-GVH-S/33 & $1080 / M$ & $+++/+++$ & 3 & 2 & ++ & ++++ & + & + & ++++ & +++ & ++ \\
\hline
\end{tabular}

*Erythema: macroscopic examination. - , Hair loss and skin erythema absent; + , moderate; ++ , marked; +++ , severe.

†Iflammation: grade determined by hematoxylin and eosin staining. Multifocal inflammatory cell infiltrate, subepithelial and hair follicle associated: grade 1, very slight; grade 2, slight; grade 3, moderate.

${ }^{\ddagger}$ Apoptosis: grade determined by hematoxylin and eosin staining. Epidermis and hair follicles with multiple apoptotic cells: - , absent; grade 2, slight.

${ }^{\S}$ MPO-positive cells (for skin, mainly dermis).

"Immunohistochemistry positive cells:,- No positive; + , single/very few (one or very few per organ slide);,++ few $(1 \%$ to $5 \%$ of cells); +++ , occasional ( $5 \%$ to $20 \%$ of cells); ++++ , frequent $(20 \%$ to $50 \%$ of cells); +++++ , very frequent ( $>50 \%$ of cells).

F, female; M, male; $C B$, cord blood; GVH-S, graft-versus-host-like signs on the skin; iDCpp65, induced-dendritic cells loaded with the pp65 viral antigen; MPO, myeloperoxidase; NRG, nonobese diabetic/Rag1 ${ }^{\text {null }} / \mathrm{IL}-2 \mathrm{R} \gamma \mathrm{c}^{\text {null }}$.

resuspended in $1 \mathrm{~mL}$ of ice-cold methanol (Th. Grayner, Renningen, Germany) and further incubated on ice for 45 minutes. Cells are washed twice with $3 \mathrm{~mL}$ of PBS (Biochrom). The cells were then resuspended in $100 \mu \mathrm{L}$ of PBS and stained with $2 \mu \mathrm{L}$ of APC-conjugated humanmouse-phospho-stat5-y694-antibody (clone SRBCZX; eBioscience, Frankfurt, Germany) for 45 minutes at room temperature in the dark. Cells are then washed twice with PBS supplemented with $0.2 \%$ of bovine serum albumin and pSTAT5 was analyzed in FACS Calibur (BD Biosciences, Heidelberg, Germany). Nontreated cells and cells treated with mouse GM-CSF were used as negative and positive controls for the assay, respectively. Data were analyzed using FlowJo and Graphpad Prism 5.01 (GraphPad Software, Inc., La Jolla, CA).

\section{Analysis of T-Cell pp65-Specific Responses by ELISP0T}

Lymphocytes recovered from lymph nodes and corresponding to $>90 \%$ human $\mathrm{T}$ cells were cryopreserved and used for functional T-cell assays, essentially as described. ${ }^{4}$ The thawed lymphocytes were first homeostatically activated with human anti-CD2/CD3/CD28-conjugated AntiBiotin MACSiBead Particles (Miltenyi Biotec) in a bead/ cell ratio of $1: 2$ and cultured in X-VIVO 15 medium for 2 days in the presence of huIL-2 (25 IU/mL) plus huIL-7 and huIL-15 (5 ng/mL each). Subsequently, T cells were further in vitro stimulated with pp65 antigen by co-culture with iDCpp65 in a DC/T cell ratio of 1:10 for additional 7 days. T cells stimulated with iDCs expressing no antigen were used as controls. For analyses of pp65-reactive IFN- $\gamma$ production, expanded $\mathrm{T}$ cells $(50,000$ cells $)$ were seeded on a 96-well ELISPOT plate coated with anti-human IFN- $\gamma$ (Mabtech, Nacka Strand, Sweden) and incubated overnight in the presence of K562 cells expressing HLA-A02*01 (heretofore artificial antigen-presenting cells or aAPC). aAPC either alone, loaded with irrelevant peptides (WT1; aAPC+WT1), pp65 overlapping peptide pool (aAPC+pp65; Miltenyi Biotec), or aAPC endogenously expressing pp65 (aAPC/pp65) were incubated with T cells expanded (see above). Twenty-four hours later, wells were washed, and a biotin-conjugated anti-human IFN- $\gamma$ monoclonal antibody was added. After incubation of plates with alkaline phosphatase-conjugated streptavidin, spots were developed using NBT/5-bromo-4-chloro-3-indolyl phosphate liquid substrate and analyzed in an AELVIS ELISPOT reader (AELVIS, Hannover, Germany).

\section{Analysis of T-Cell pp65-Specific Responses by Intracellular Staining}

T cells recovered from lymph nodes (LNs) were also analyzed by intracellular staining for detection of antigen-specific IFN- $\gamma$ secretion, as described. ${ }^{4}$ As a negative control for gating, nonstimulated lymphocytes were used. $\mathrm{T}$ cells expanded homeostatically and antigenically were stimulated for 16 hours with $10 \mu \mathrm{g} / \mathrm{mL}$ of CMV PepTivator (pp65 overlapping peptide pool; Miltenyi Biotec). The Wilms tumor 1 (WT1) overlapping peptide pool (Miltenyi Biotec) was used as negative control for peptide stimulation. Protein transport inhibitor cocktail (eBioscience, Frankfurt, Germany) was added to the cells 2 hours after stimulation. After 16 hours, $\mathrm{T}$ cells were harvested, stained with APC-conjugated anti-human CD3, APC$\mathrm{H} 7$ conjugated CD4, and PCy7-conjugated anti-human CD8 antibodies (Biolegend). After fixation/permeabilization with Cyofix/perm (BD Biosciences) for 20 minutes at $4^{\circ} \mathrm{C}$ and washing, anti-human phosphatidylethanolamine-IFN- $\gamma$ (eBioscience) was used for staining for 30 minutes. The cells were acquired by flow cytometry using LSRII (BD Biosciences) and analyzed by FlowJo software. 
A

Control

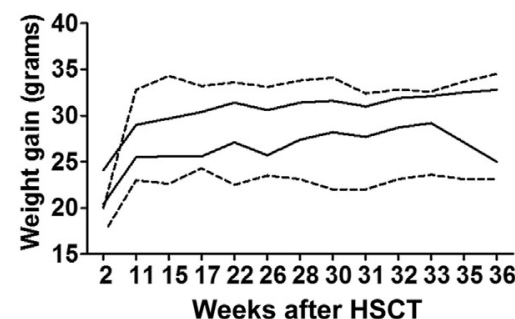

Merged data CB $1+2$
Control

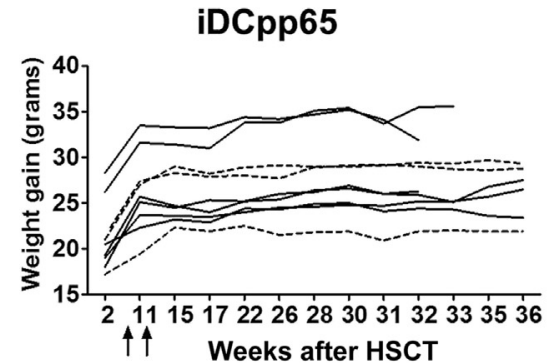

Weeks after HSCT

\section{- $\mathrm{CB} 1$}

--. CB 2
CB 1

iDCpp65

iDCpp65, GVH-S

$B$ huCD45+ in PBL

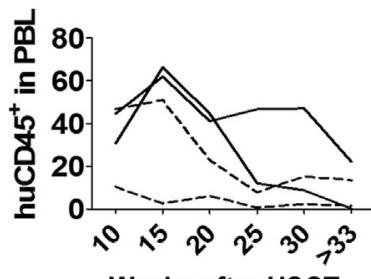

Weeks after HSCT

C $\mathrm{CD}^{+}$in $\mathrm{CD}^{+} 5^{+}$
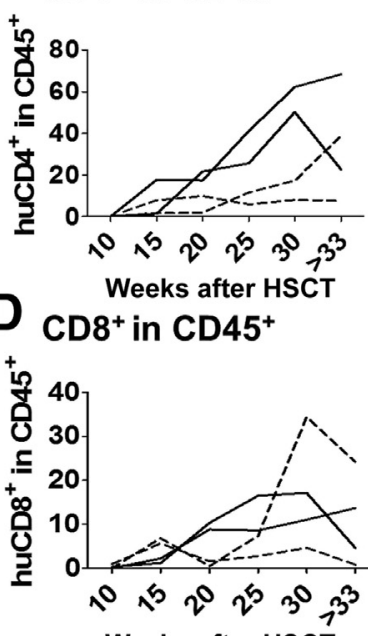

E CD19 $^{+}$in $\mathrm{CD}^{+} 5^{+}$

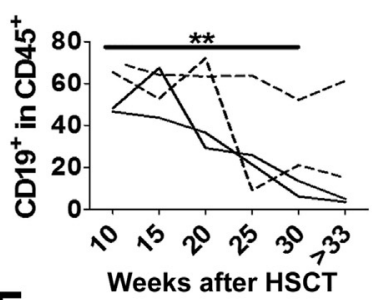

F $\mathrm{CD}^{+} 4^{+}$in $\mathrm{CD}^{+} 5^{+}$

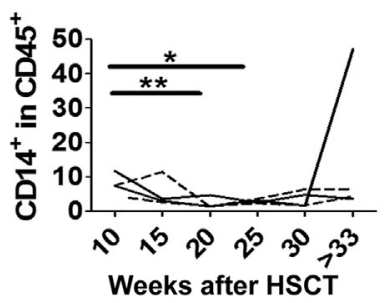

iDCpp65
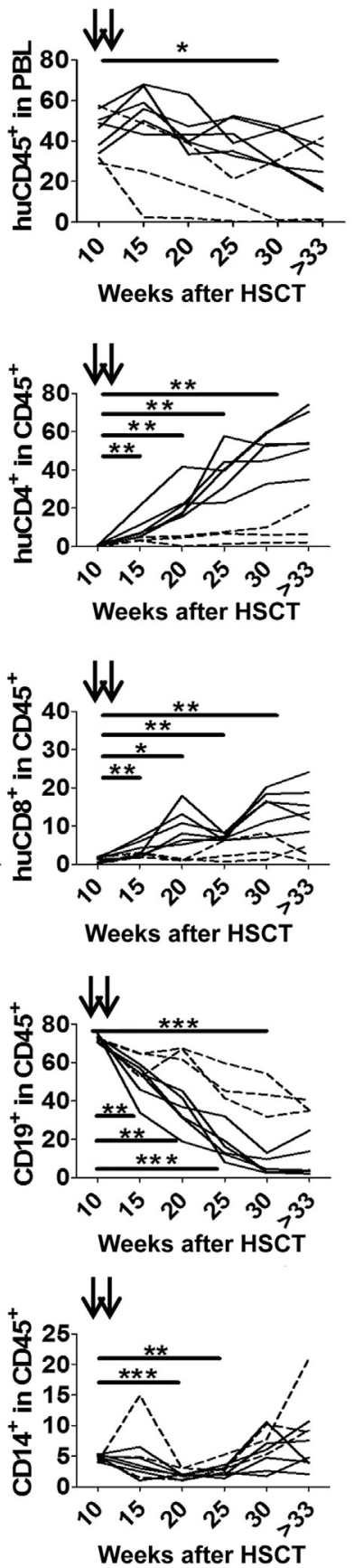
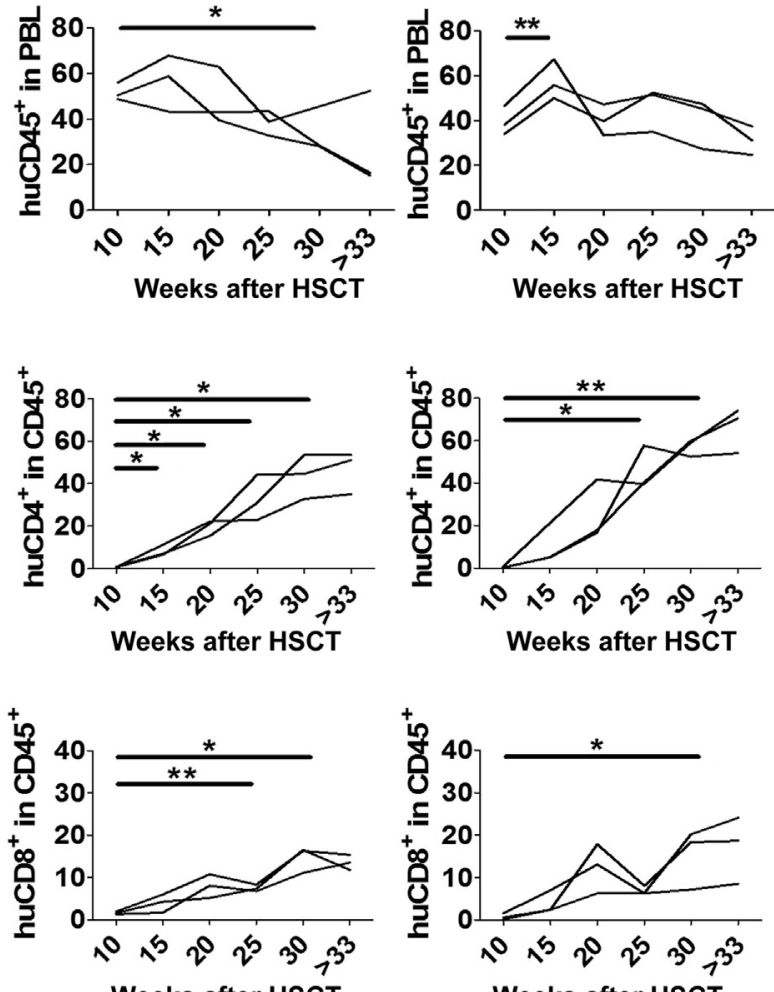

Weeks after HSCT
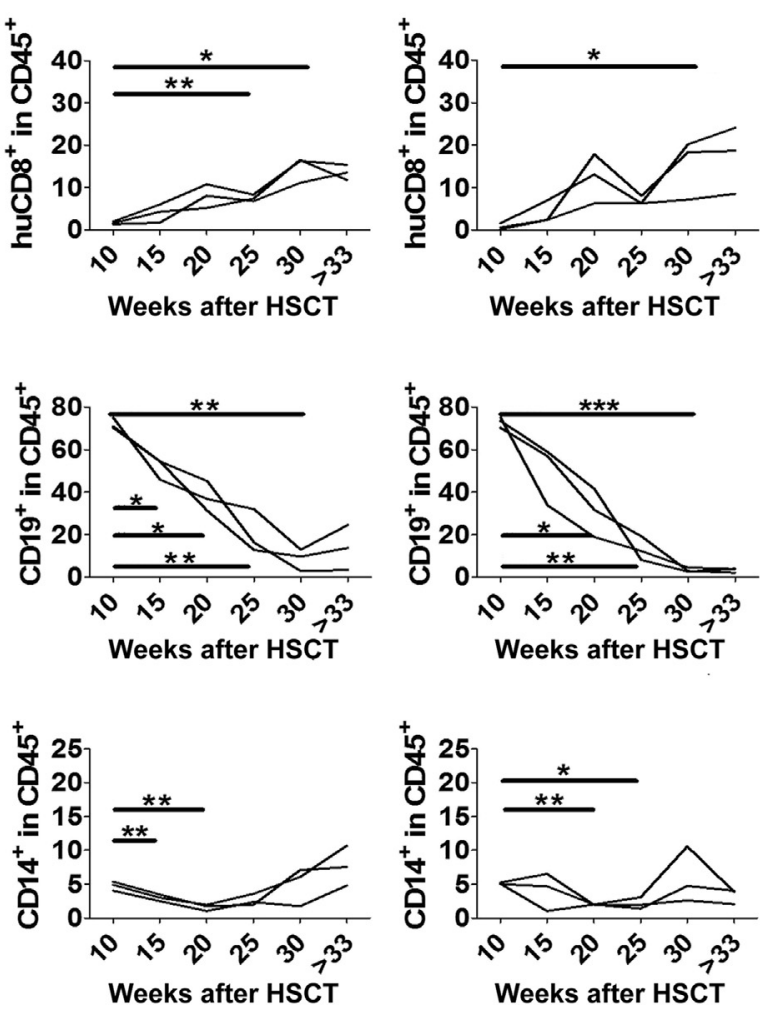


\section{Statistical Analysis}

Simulating the analyses that would be applicable for patients in a clinical trial after HSCT, statistical analyses were performed for each cohort (control versus iDCpp65 immunization) comparing the immune reconstitution between the baseline at 10-week after HSCT time point (confirming hematopoietic engraftment before iDCpp65 immunization) and subsequent time points (at 15,20, 25, and 30 weeks after HSCT; statistical analyses were not considered after 30 weeks because of the skin pathology observed). For this purpose, paired $t$-test of unequal variances was performed to compare the differences among groups for engraftment of human hematopoietic lineages in blood of NRG mice. Unpaired $t$-test of unequal variances was performed to compare the differences among groups for engraftment of human hematopoietic lineages in organs of NRG mice and for the cytokines detected in mice plasma. A two-way analysis of variance was performed to compare the mean differences of the IFN- $\gamma$ ELISpot assay using $\mathrm{T}$ cells explanted from lymph nodes (LN and LN/GVH-S) that were stimulated with iDCs and iDCpp65 in vitro. All tests were two sided and performed at a significance level of 0.05. Data were analyzed with GraphPad Prism software version 7.

\section{Results}

Long-Term Persistency of Human Cells in NRG Mice after CD34 ${ }^{+}$Cell Transplantation and iDCpp65 Immunization

NRG mice were transplanted with purified $\mathrm{CD} 34^{+}$cells from two different cord blood units (CB1, eight mice; CB2, five mice). Subsequently, prime/boost immunizations with iDCpp65 were administered at weeks 10 and 11 after CB-HSCT (Figure 1A). Transplanted mice were randomly allocated into immunized and nonimmunized cohorts (Table 2). $\mathrm{CD}^{+} 4^{+}$and $\mathrm{CD} 14^{+}$cells showed a high purity $>95 \%$ (Figure 1B), which was important to avoid GVH disease (GVHD) caused by contaminating human $\mathrm{T}$ cells. iDCpp65 was generated with $\mathrm{CD} 14^{+}$monocytes transduced with the IDLV coexpressing GM-CSF, IFN- $\alpha$, and pp65 and cryopreserved. After thawing, transduction efficiency was confirmed by detectable IDLV copies (six to seven copies per cell) (Figure 1C). In vitro culture of the thawed cells for 7 days and flow cytometry analyses confirmed the identity of iDCpp65: down-regulation of CD14 and up-regulation of CD80, HLA-DR, and CD86 (Figure 1D). A total of $5 \times 10^{5}$
iDCpp65s were injected s.c. bilaterally near the inguinal lymph nodes $\left(1 \times 10^{6}\right.$ iDCpp65 viable cells $)$. All mice gained weight normally (Figure 2A) and showed no signs of wasting syndrome, a clinical sign generally associated with systemic GVHD. All control mice (CB1, $n=2$; CB2, $n=2)$ and six of nine iDCpp65-immunized mice (CB1, $n=3$; CB2, $n=3$ ) showed no clinical signs of pathologies for up to 30 weeks after HSCT. Nevertheless, 30 weeks after HSCT, three mice transplanted with CB1 started showing signs of severe skin inflammation and erythema, indicating a GVH-like effect in skin (which we called GVHS), and were sacrificed at 33 weeks after HSCT (Table 2). The frequencies of human hematopoietic cell populations in PBL were compared between the immunized and control cohorts (Figure 2, B-F, and Supplemental Table S1). Mice transplanted with CB1 and immunized with iDCpp65 were compared in subcohorts without erythema versus with GVH$\mathrm{S}$. The frequency of huCD $45^{+}$cells in PBL was generally lower for the iDCpp65-immunized group $(P<0.05$ at 30 weeks after HSCT). Nevertheless, within the huCD $45^{+}$cells, the frequencies of $\mathrm{CD}^{+} \mathrm{T}$ helper and $\mathrm{CD}^{+} \mathrm{T}$ cytotoxic lymphocytes after iDCpp65 immunization were always considerably higher relative to baseline levels, whereas the same was not observed for the control cohort (Figure 2, C and D, and Supplemental Table S1). Concurrent with the increases of the T-cell frequencies, continuous decreases in the frequencies of human $\mathrm{CD} 19^{+} \mathrm{B}$ cells were observed for all time points of the iDCpp65-immunized cohort (Figure 2E and Supplemental Table S1). The frequencies of the human $\mathrm{CD} 14^{+}$monocytes also generally decreased for both control and iDCpp65 immunized cohorts, considerably at 20 and 25 weeks after HSCT (Figure 2F and Supplemental Table S1). Comparisons between the subcohorts of CB1 immunized with iDCpp65 showed that the mice without GVH-S initially had comparable growth kinetic of $\mathrm{CD}^{+}{ }^{+} \mathrm{T}$ cells until 20 weeks after HSCT; however, at 25 and 30 weeks after HSCT, mice with GVH-S started to show a higher rate of $\mathrm{CD} 4^{+} \mathrm{T}$ cell growth relative to baseline in comparison with immunized mice without GVH-S pathology (Figure 2C and Supplemental Table S2). These results corroborated with our previous observations obtained with a shorter, 16-week postHSCT model, ${ }^{6}$ showing that iDCpp65 immunization can augment the human T-cell reconstitution (particularly of $\mathrm{CD}^{+}{ }^{\mathrm{T}}$ cells) in humanized mice. In addition, the subcohort of mice with GVH-S showed a higher increase in the frequencies of $\mathrm{CD}^{+} \mathrm{T}$ cells in PBL at the onset of the pathology than the subcohort of mice not developing severe skin inflammation.

Figure 2 Weight monitoring and analyses of immune reconstitution in peripheral blood of each individual mouse. Arrows indicate time point of iDCpp65 administrations. A: Weight monitoring of control and iDCpp65-immunized mice. B: Longitudinal analyses of human chimerism measured by monitoring the frequencies of human $\mathrm{CD} 45^{+}$hematopoietic cells in peripheral blood. $\mathbf{C}-\mathbf{F}$ : Longitudinal analyses of frequencies of CD4 ${ }^{+} \mathrm{T}$ cells $(\mathrm{C}), \mathrm{CD} 8^{+} \mathrm{T}$ cells $(\mathrm{D}), \mathrm{CD} 19^{+}$ $(\mathbf{E})$, and $\mathrm{CD}_{14}{ }^{+}(\mathbf{F})$ cells within the human $\mathrm{CD} 45^{+}$cells. A paired $t$-test was applied to evaluate the mean difference between week 10 and subsequent time points (excluding week $>33$ ) for each group. $n=4$ (A, control mice); $n=9(\mathbf{A}$, iDCpp65-immunized mice); $n=4(\mathbf{B}-\mathbf{F}, \mathbf{C B} 1+2$, control); $n=9(\mathbf{B}-\mathbf{F}$, CB1+2, iDCpp65); $n=3$ (B-F, CB1, iDCpp65); $n=3$ (B-F, CB1, iDCpp65/GVH-S). ${ }^{*} P<0.05,{ }^{*} P<0.01$, and ${ }^{* *} P<0.001$. HSCT, hematopoietic stem cells transplantation. 
Mice Immunized with iDCpp65 Show Higher Proportion of $\mathrm{CD}^{+}$and $\mathrm{CD}^{+}$Effector Memory Subtypes among Other T-Cell Subtypes in PBL

Another previously documented effect of the iDCpp65 immunization in humanized mice was the activation of naïve (N) and CM T cells toward terminally differentiated EM T cells. ${ }^{6}$ Therefore, we compared the frequencies of $\mathrm{N}\left(\mathrm{CD} 45 \mathrm{RA}^{+} \mathrm{CD}^{2} \mathrm{~L}^{+}\right), \mathrm{CM}\left(\mathrm{CD} 45 \mathrm{RA}^{-} \mathrm{CD}^{-} \mathrm{L}^{+}\right)$, and EM (CD45RA $\left.{ }^{-} \mathrm{CD}^{2} \mathrm{~L}^{-}\right)$T-cell subtypes relative to the total $\mathrm{T}$ cells between the control and iDCpp65 immunized cohorts (Figure 3 and Supplemental Table S3). As a baseline, we used values measured at week 15 after HSCT, when the human $\mathrm{T}$-cell development in mouse thymus and emigration to the PBL were clearly measurable. ${ }^{4}$ Corroborating with our previous results, ${ }^{4}$ the effects of iDCpp65 affected predominantly the development of $\mathrm{EM} \mathrm{CD} 4^{+}$and EM CD8 ${ }^{+} \mathrm{T}$ cells. In this current, single prime-boost immunization model, the iDCpp65 immunizations resulted in lower frequencies on CM CD4+ cells (Figure 3A), whereas the iDCpp65 immunization effects on the increased frequencies of $\mathrm{CD}^{+} \mathrm{EM} \mathrm{T}$ cells were substantial at 25 $(P=0.001)$ and 30 weeks $(P=0.004)$ after HSCT (Figure 3B and Supplemental Table S3). The effects of iDCpp65 immunization were also substantial for $\mathrm{CD} 8^{+} \mathrm{EM}$ T cells $25(P=0.003)$ and 30 weeks $(P=0.008)$ after HSCT (Figure 3D). Regarding the comparison between mice transplanted with CB1 without and with GVH-S, the only significant difference was observed at week 30 after HSCT, when the increase in the frequency of EM CD4 $4^{+}$ $\mathrm{T}$ cells relative to week 15 was higher for the GVH-S cohort than for mice with no pathology $(P=0.024$ and $P=0.003$, respectively) (Figure $3, \mathrm{~B}$ and $\mathrm{C}$, and Supplemental Table S4). Thus, the levels of activated EM $\mathrm{CD} 4^{+} \mathrm{T}$ cells increased at the onset of GVH-S.

\section{Mice with Inflammation and GVH-S Show Abundant Intrafollicular $\mathrm{CD}^{+}$Cells Surrounded by Mouse Macrophages in Dermis}

A systemic GVH disease could explain why three of six mice transplanted with CB1 and immunized with iDCpp65 showed severe skin inflammation on the head and on the back. This was diagnosed as a skin redness and inflammation, particularly on the head and around eyes, and hair loss (Figure 4A). Because exacerbation of GVH would be a highly detrimental effect for patients after HSCT, we performed extensive histopathological analyses of several organs to characterize the pathology in this nonclinical model (Figure 4, B-D, Table 2, and Supplemental Table S5). Because mice with GVH-S did not lose weight and did not show any inflammatory reaction in the colon (data not shown), this indicated rather a local immune reaction provoked by the human immune system to the mouse skin instead of systemic pathology. Hematoxylin and eosin staining revealed multifocal slight to moderate inflammatory cell infiltrate in the subepithelial region, associated with dermis and hair follicles. Epidermis and hair follicles showed multifocal very slight to slight apoptotic cells. Periodic acid-Schiff stain indicated no fungi or parasites (data not shown). Giemsa staining showed infiltrations with single eosinophils and occasional mast cells (data not shown). We performed a series of immunohistochemistry analyses and scoring of the affected skin areas (Table 2 and Supplemental Table S5). Staining with anti-human nuclei antibody showed no signal for NRG nontransplanted mice (Supplemental Table S5). On the other hand, Hu-HSCT mice revealed multifocal (occasional to frequent) positive nuclei in all humanized mice, which was associated with the detection of frequent HLA-DR ${ }^{+}$cells (Supplemental Table S5). In general, just a few human macrophages (detected with anti-human CD68) and human dendritic cells (detected with human anti-Langerin, anti-CD1a, and anti-CD11c) were detected in the dermis and epidermis of humanized mice (Figure 4B and Supplemental Table S5). On the other hand, large clusters of human $\mathrm{CD}^{+}$and $\mathrm{CD}^{+}$positive lymphocytes could be frequently observed, mainly associated with basal cells of hair follicles and in the epidermis and dermis in the skin of iDCpp65-immunized mice (Figure 4B, Table 2, and Supplemental Table S5), whereas human $\mathrm{CD}^{+} \mathrm{T}$ cells, human B cells (stained for CD79 $\alpha$ and CD20), human mast cells (tryptase ${ }^{+}$), and granulocyte $\left(\right.$ myeloperoxidase ${ }^{+}$) cells were infrequently detected (Supplemental Table S5). Erythroblasts were not at all detected with anti-human glycophorin staining (data not shown). On the other hand, mouse macrophages detected after staining against F4/80 showed occasional to frequent positive cells next to basal cells of hair follicles, in epidermis, dermis, and subcutis. The iDCpp65/GVH-S group showed marked infiltration of mouse macrophages in the skin (Figure 4, C and D, and Supplemental Table S5). Altogether, these results indicated that the interaction between human $\mathrm{CD} 4^{+} \mathrm{T}$ cells and mouse macrophages was linked to the skin inflammation and GVH-S.

\section{Mice with GVH-S Also Show Massive Expansion and Clustering of $\mathrm{CD}^{+}{ }^{+} \mathrm{T}$ Cells in Spleen}

Spleen sections from control and iDCpp65-immunized mice were stained and compared (Figure 5). Human cells (antihuman nuclei ${ }^{+}$) typically localized sparsely in spleen areas of the control nonimmunized mice, but formed large clusters around the central arterioles of iDCpp65-immunized and iDCpp65/GVH-S mice (Figure 5 and Table 2). Immunostaining of serial spleen sections of these mice for F4/80 showed that mouse macrophages surrounded the human $\mathrm{CD} 4^{+} \mathrm{T}$-cell clusters, whereas in control nonimmunized mice they were randomly distributed (Figure 5). Inside reconstituted lymph nodes, human $\mathrm{CD}^{+}{ }^{+} \mathrm{T}$ cells were abundant and disorganized, surrounded by a thin layer of mouse macrophages in the capsular zone (Supplemental Figure S1). 


\section{Cord blood $1+2$}

\section{Control}

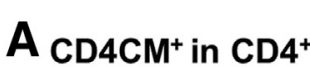

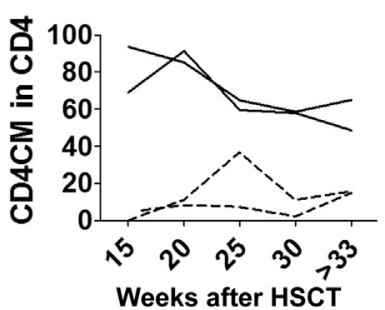

\section{$B$ CD4EM ${ }^{+}$in $\mathrm{CD}^{+}$}

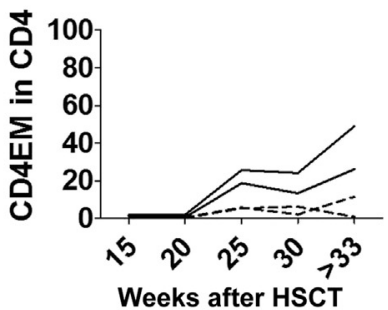

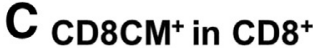

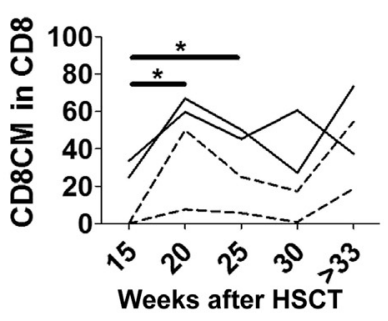

\section{D $\mathrm{CD}^{2} \mathrm{EM}^{+}$in $\mathrm{CD}^{+}$}

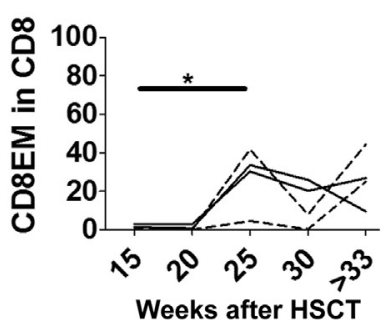

iDCpp65
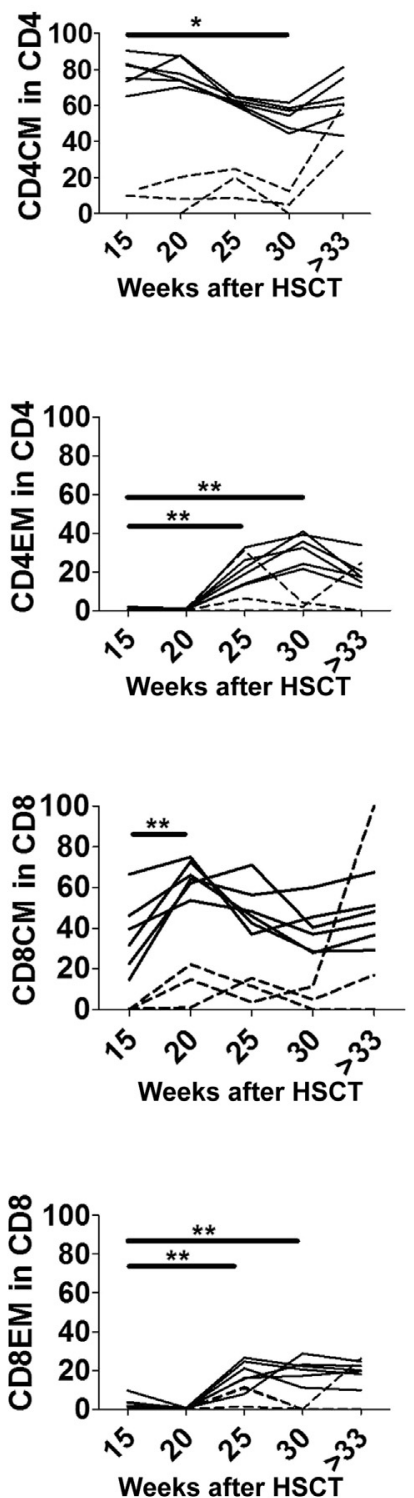

\section{Cord blood 1}

iDC-CB1

iDC-CB1, GVH-S
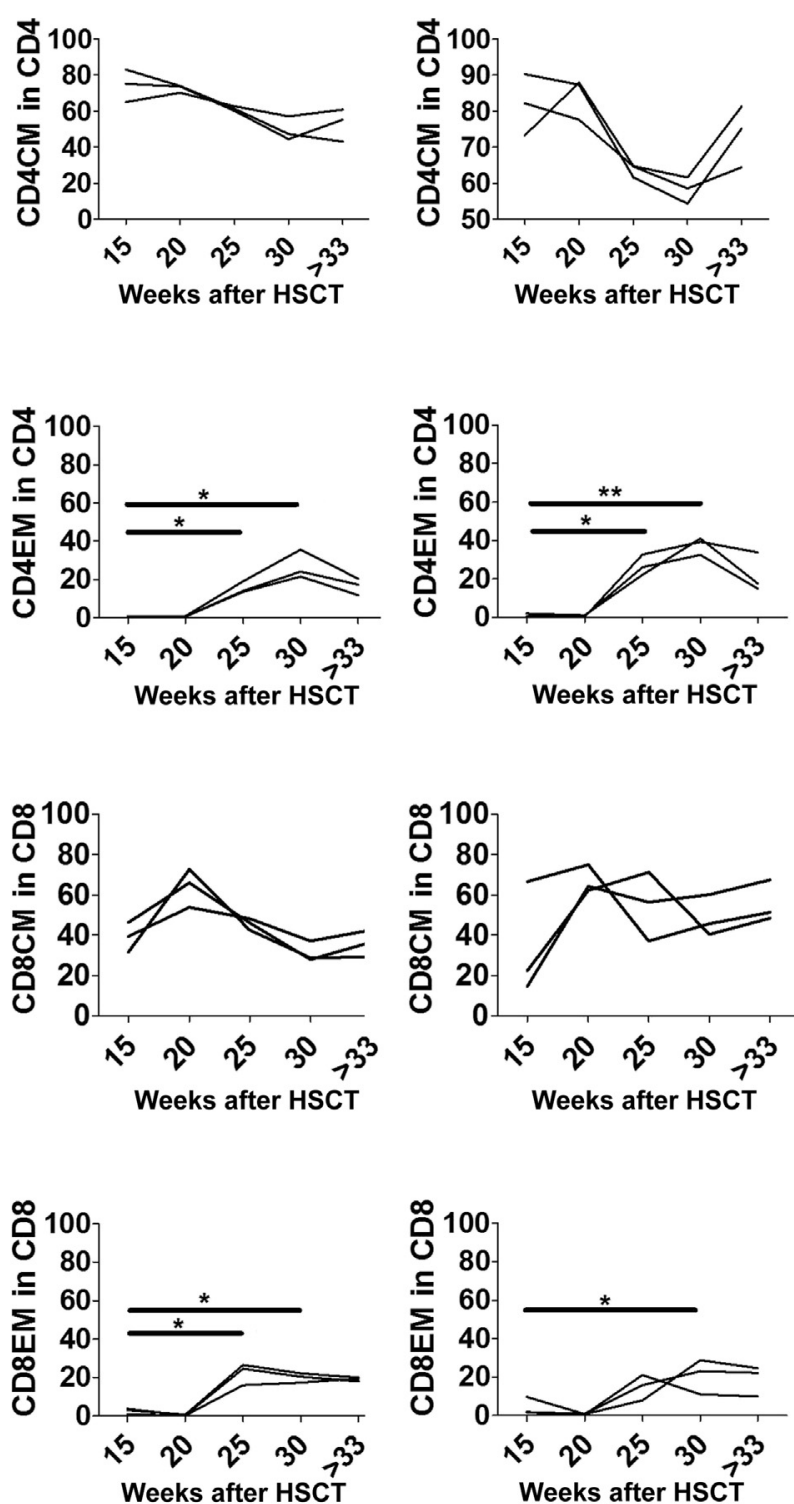
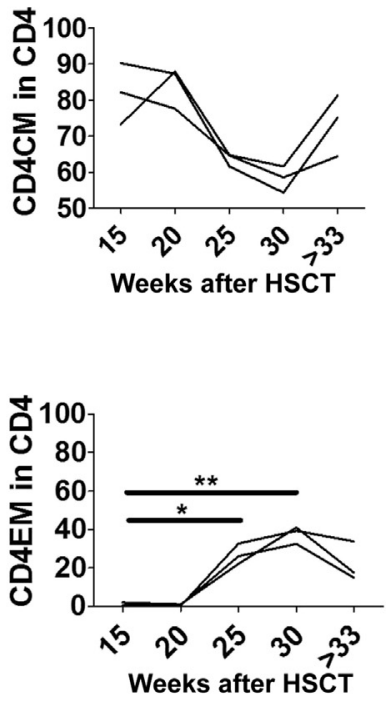

Weeks after HSCT

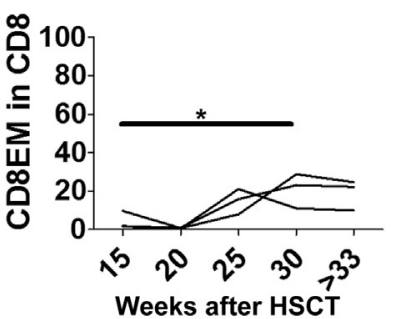

Figure 3 Longitudinal analyses of human T-cell subtypes in peripheral blood of each individual mouse. The proportion of CD4 CM (A) and CD4 EM (B) cells within total $\mathrm{CD}^{+}$cells measured at different time points. The proportion of CD8 CM (C) and EM (D) cells within total CD8 ${ }^{+}$cells measured at different time points. A paired $t$-test was applied to evaluate the mean difference between week 15 and subsequent time points (excluding week $>33$ ) for each group. $n=4$ (CB1+2, control); $n=9$ (CB1+2, iDCpp65); $n=3$ (iDC-CB1); $n=3$ (CB1, iDC-CB1, GVH-S). ${ }^{*} P<0.05,{ }^{* *} P<0.01$. HSCT, hematopoietic stem cells transplantation.

Increased Absolute Numbers of Human Mature T Cells in Spleen and Lymph Nodes of iDCpp65-Immunized Mice with GVH-S

Lymphocytes recovered from spleen, peripheral LNs, and mesenteric LNs were analyzed by flow cytometry. As a general trend, the numbers of huCD45 ${ }^{+}, \mathrm{CD} 4^{+}$, and $\mathrm{CD} 8^{+}$ $\mathrm{T}$ cells in spleen and peripheral LNs of CB1 reconstituted mice were higher for mice immunized with iDCpp65 developing GVH-S in comparison to control and immunized mice (Figure 6, A and D, and Supplemental Table S6). Remarkably, the inverse condition was observed for the repopulation of the mesenteric lymph nodes. Mice with GVH-S showed the lowest numbers of human lymphocytes in the colon (Figure $6 \mathrm{G}$ ), possibly because they were largely recruited to the skin. Analyses of the $\mathrm{T}$ cells repopulating 
A

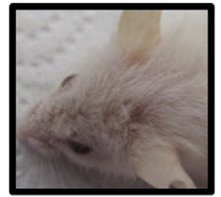

Control

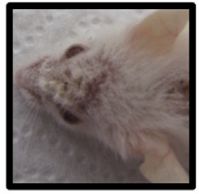

iDCpp65

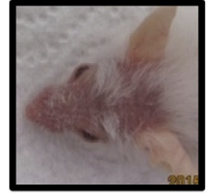

iDCpp65/ GVH-S
B

H\&E

F4/80

AHN

CD3

CD4

CD8

CD68

CD1a
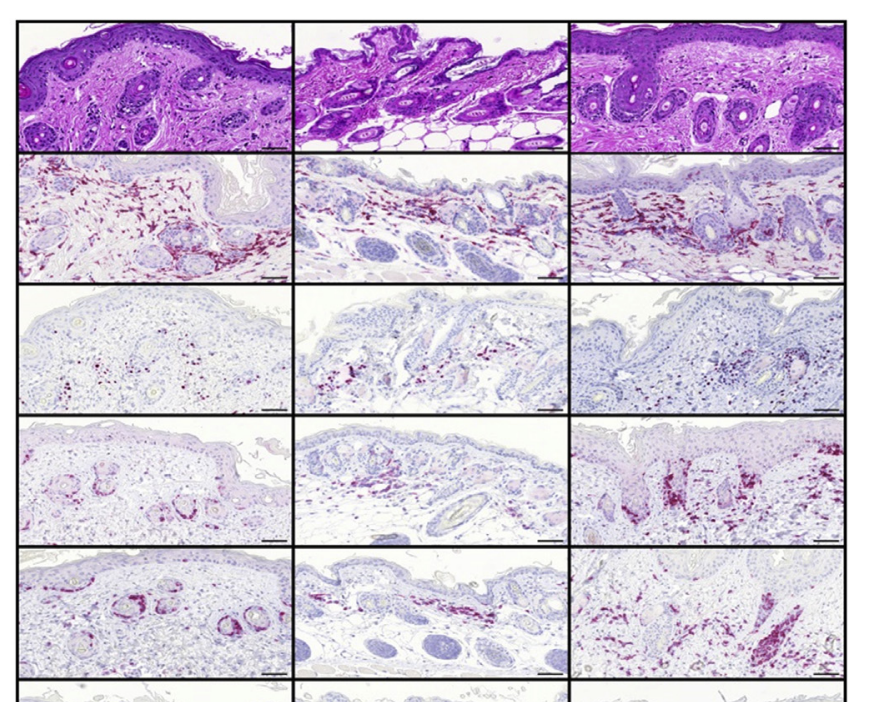

C

CD4

F4/80
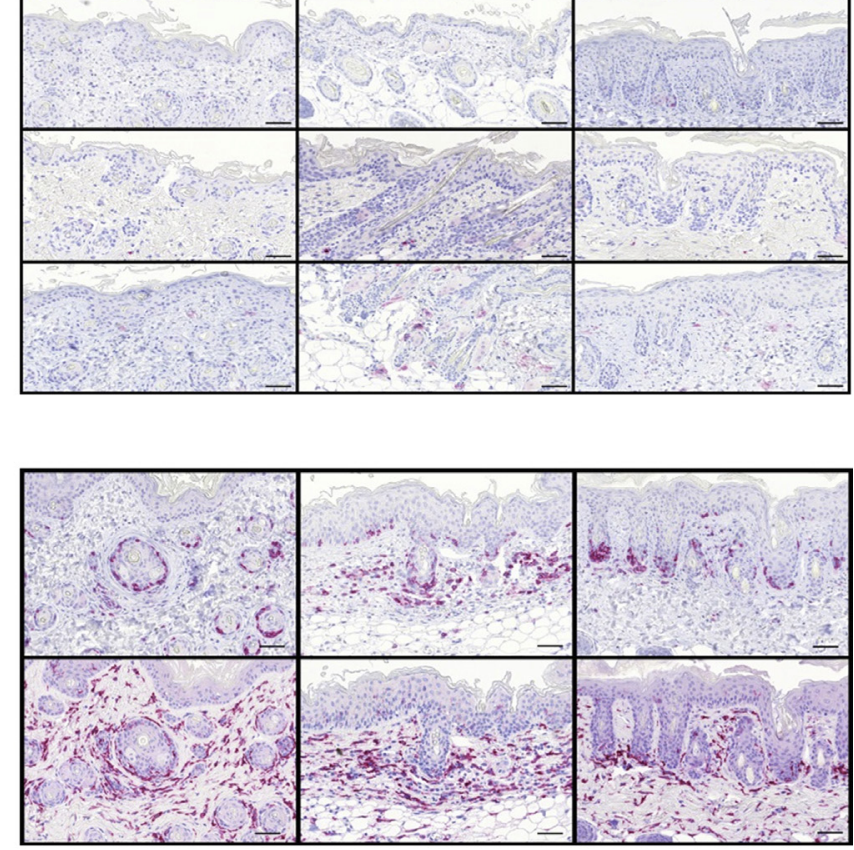

D

CD4

F4/80

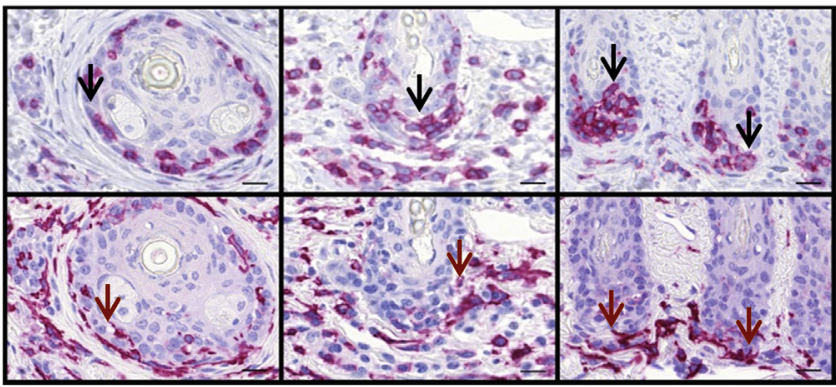

Figure 4 Mice reconstituted with CB1 show skin inflammation that was assessed by postmortem histopathology analyses. A: Macroscopic observation. Macroscopic examination showing GVH-S on the head of representative iDCpp65 immunized mice. B: Histopathological analyses of skin showing the baseline distribution of cell populations for representative mice corresponding to nonimmunized control mouse 36 weeks after transplant, iDCpp65-immunized mouse 36 weeks after transplant, and iDCpp65-immunized mouse affected with GVH-S and sacrificed 33 weeks after transplant. Hematoxylin and eosin (H\&E) staining and immunohistochemical detection of the mouse macrophage marker (F4/80), anti-human nuclei (AHN), anti-human CD3, anti-human CD4, antihuman CD8, anti-human CD68, and anti-human CD1a are shown. C and D: Histopathological analyses of skin showing in higher magnification the histopathological analyses of skin of a representative mouse for each cohort. Serial sections were stained immunohistochemically for detection of the human CD4 mouse macrophages (F4/80). In all groups, $\mathrm{CD}^{+}$cells are primarily observed in the basal cell areas and in the neighborhood of hair follicles. Detail enlargements show CD4-positive cells in basal cell layers of hair follicles (black arrows). F4/80-positive mouse macrophages are seen in the perifollicular regions and the connective tissue (red arrows). Scale bars: $50 \mu \mathrm{m}$ (B and C); $15 \mu \mathrm{m}$ (D). 
Control

H\&E

F4/80

AHN

CD4

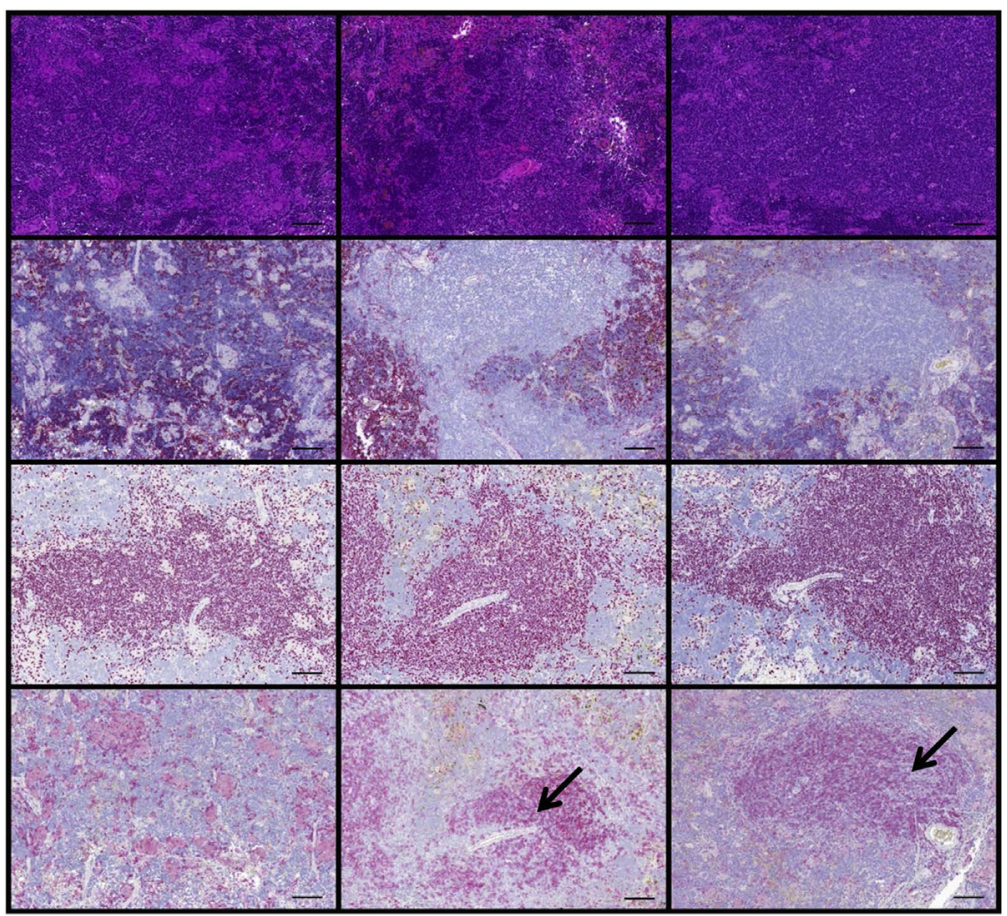

Figure 5 Analyses of mouse macrophages and human $\mathrm{CD}^{+}{ }^{+} \mathrm{T}$ cells in the spleen. Histopathological analyses of the spleen showing the baseline distribution of cell populations for representative mice corresponding to nonimmunized control mouse 36 weeks after transplant, iDCpp65immunized mouse 36 weeks after transplant, and iDCpp65-immunized mouse affected with GVH-S and sacrificed 33 weeks after transplant. Hematoxylin and eosin (H\&E) staining, immunohistochemical detection of the mouse macrophage marker (F4/80), the anti-human nuclei (AHN), and the human CD4 marker are shown. Arrows show mouse macrophages surrounding large clusters of human $\mathrm{CD4}^{+} \mathrm{T}$ cells in the spleen of iDCpp65immunized mice. Scale bars $=100 \mu \mathrm{m}$. the spleen and peripheral LNs revealed an abundance of

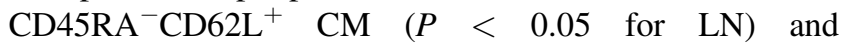
$\mathrm{CD} 4 \mathrm{RA}^{-}{ }^{-}$CD62L EM CD4 ${ }^{+}{ }^{\mathrm{T}}$ cells (Figure 6, E, F, H, and I). In contrast, lower numbers of memory $\mathrm{CD}^{+} \mathrm{T}$ cells were detected in mesenteric LNs (Figure 6, B and E). The same trend was observed for $\mathrm{CM}$ and $\mathrm{EM} \mathrm{CD} 8^{+} \mathrm{T}$ cells (Figure 6, C, F, and I). Thus, interestingly, iDCpp65immunized mice with GVH-S seemed to be the best responders in terms of accumulation of mature human $\mathrm{T}$ cells in spleen and peripheral lymph nodes. Lack of activated human $\mathrm{T}$ cells in the mesenteric lymph nodes was in line with no inflammation detectable in the colon of mice with GVH-S (data not shown).

\section{Plasma from iDCpp65-Immunized Mice with GVH-S Shows Low Levels of Mouse Cytokines and High Levels of Human Th2-Type Cytokines}

We measured the concentrations of murine and human GMCSF, MCP-1, IL-6, IL-8, IL-10, IFN- $\gamma$, IL-5, IL-12p70, and tumor necrosis factor- $\alpha$ cytokines in the plasma of humanized mice (detection limit for the assay $>10 \mathrm{pg} / \mathrm{mL}$; some data points were extrapolated) (Figure 7A). For GM-CSF, which is a key growth factor for macrophage activation, the cytokine detected was predominantly human, with significantly higher levels detected in iDCpp65-immunized mice with GVH-S compared with less affected iDCpp65 mice $(P=0.04$; average, $500 \mathrm{pg} / \mathrm{mL}$ ). This was surprising because human GM-CSF is not supposed to cross-activate mouse cells.
Therefore, most likely for the mice with GVH-S, human GMCSF might have participated in the activation mouse (and not human) macrophages. The same pattern was also observed for other relevant human Th2-type cytokines: MCP-1 $(P>0.05$; average, $600 \mathrm{pg} / \mathrm{mL})$, IL-6 $(P=0.034$; average, $150 \mathrm{pg} / \mathrm{mL}), \mathrm{IL}-8(P=0.039$; average, $20 \mathrm{pg} / \mathrm{mL})$, and IL-10 $(P>0.05$; average, $40 \mathrm{pg} / \mathrm{mL})$. Remarkably, although these human cytokines were all elevated in mice with GVH-S, the equivalent mouse cytokines were dramatically reduced. This shift toward a human Th2-type cytokine pattern was not absolute, as human IFN- $\gamma$ (which is typically produced by Th1-type CD ${ }^{+}$T cells, cytotoxic T lymphocytes, and natural killer cells) was also elevated in iDCpp65/GVH-S mice ( $P=0.033$; average, $600 \mathrm{pg} / \mathrm{mL})$. On the other hand, other Th1-type human cytokines analyzed (IL-5, IL-12p70, and tumor necrosis factor- $\alpha$ ) were not altered in iDCpp65/GVH-S mice (Figure 7B). Therefore, these results supported the notion that a mixture of human, but not mouse, cytokines were involved with the immune responses and skin pathology involving mouse macrophages.

\section{Cross-Reactivity of Human Th2-Type Cytokines with Mouse Macrophages}

High levels of cytokines in the plasma can lead to several types of pathologies and, in excess, to cytokine release syndrome (CRS). ${ }^{8} \mathrm{CRS}$ is generally known as non-antigenassociated toxicity, which occurs because of a high level of T-cell immune activation. In our study, mice presenting 


\section{$\bigcirc$ Control iDCpp65 $\triangle$ iDCpp65/GVH-S}

A

\section{Lymphocytes}

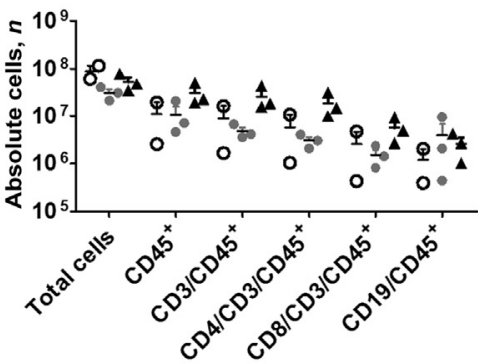

D

\section{Lymphocytes}

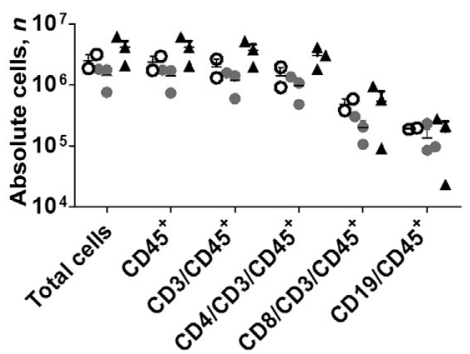

G

\section{Lymphocytes}

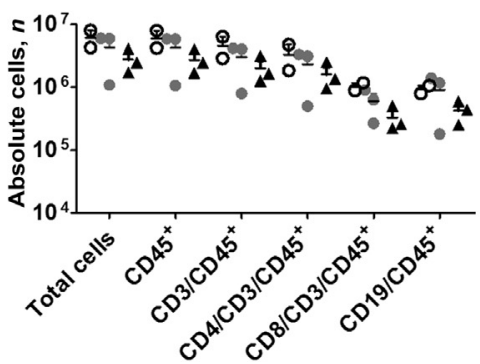

Spleen

$\mathrm{CD4}^{+}$immunophenotypes

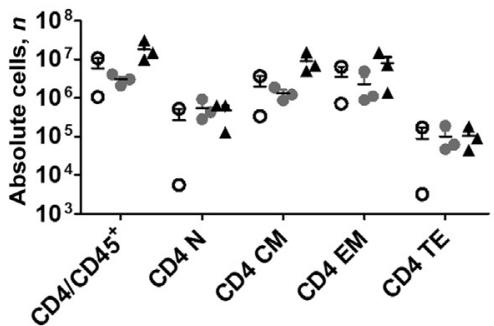

\section{Lymph nodes}

E $\mathrm{CD}^{+}$immunophenotypes

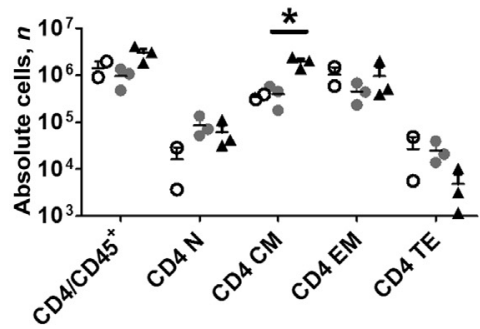

\section{Mesenteric lymph nodes}

H $\mathrm{CD}^{+}$immunophenotypes

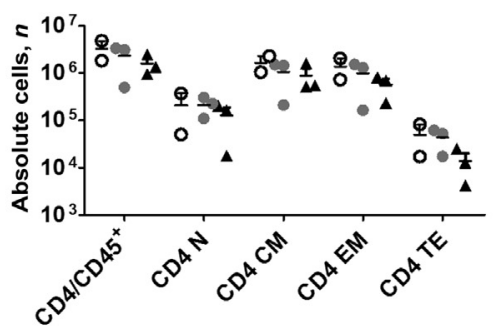

C $\mathrm{CD8}^{+}$immunophenotypes
F $\mathrm{CD}^{+}{ }^{+}$immunophenotypes

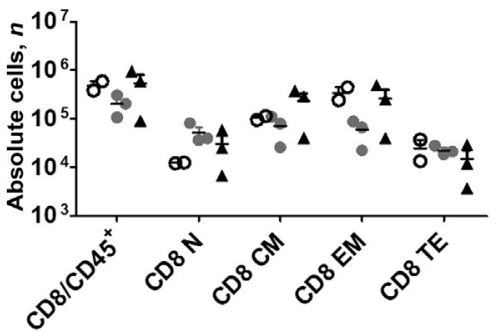

Figure 6 Flow cytometry analyses for quantification of human lymphocytes in spleen, lymph nodes, and mesenteric lymph nodes of each mouse reconstituted with CB1 at post-mortem analyses (33 to 36 weeks after transplantation). Quantification of total cells in tissue, huCD45 ${ }^{+}, \mathrm{CD}^{+}{ }^{+}$in huCD45 $5^{+}, \mathrm{CD} 4^{+}$in huCD45 $5^{+}, \mathrm{CD}^{+}$in huCD45 $5^{+}$, and $\mathrm{CD} 19^{+}$in huCD45 ${ }^{+}$for spleen, lymph node, and mesenteric lymph nodes, respectively (A, D, and $\left.\mathbf{G}\right)$. Quantification of CD4 ${ }^{+}$in

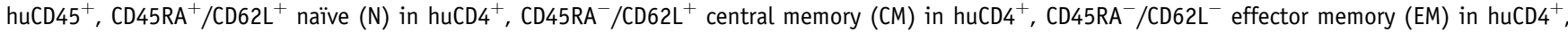
$\mathrm{CD} 5 \mathrm{RA}^{+} / \mathrm{CD}_{2} \mathrm{~L}^{-}$terminal effector (TE) in huCD4 ${ }^{+}$, for spleen, lymph node, and mesenteric lymph nodes, respectively $(\mathbf{B}, \mathbf{E}$, and $\mathbf{H})$. Quantification of CD8 ${ }^{+}$in huCD45 $5^{+}, \mathrm{N}$ in huCD8 ${ }^{+}, \mathrm{CM}$ in huCD8 $8^{+}, \mathrm{EM}$ in huCD8 ${ }^{+}, \mathrm{TE}$ in huCD8 ${ }^{+}$, for spleen, lymph node, and mesenteric lymph nodes, respectively (C, $\mathbf{F}$, and $\left.\mathbf{I}\right)$. Statistical significance comparing iDCpp65-immunized mice without and with GVH-S is indicated on the graphs. Trend with $P=0.08$ was observed for CD4 ${ }^{+}$in huCD45 $5^{+}$in lymph nodes. ${ }^{*} P<0.05$.

GVH-S showed consistently high activation of human $\mathrm{CD}^{+}{ }^{+} \mathrm{T}$ lymphocytes, high levels of human Th2-type cytokines, and activated mouse macrophages (F4/80). Therefore, to mimic the activation of mouse macrophages with human cytokines, we stimulated the RAW264.7 mouse macrophage cell line with human MCP-1, IL-6, IL-8, and IL-10 in various combinations in vitro (Figure $7 \mathrm{C}$ ). Unstimulated RAW264.7 cells or cells stimulated with murine 


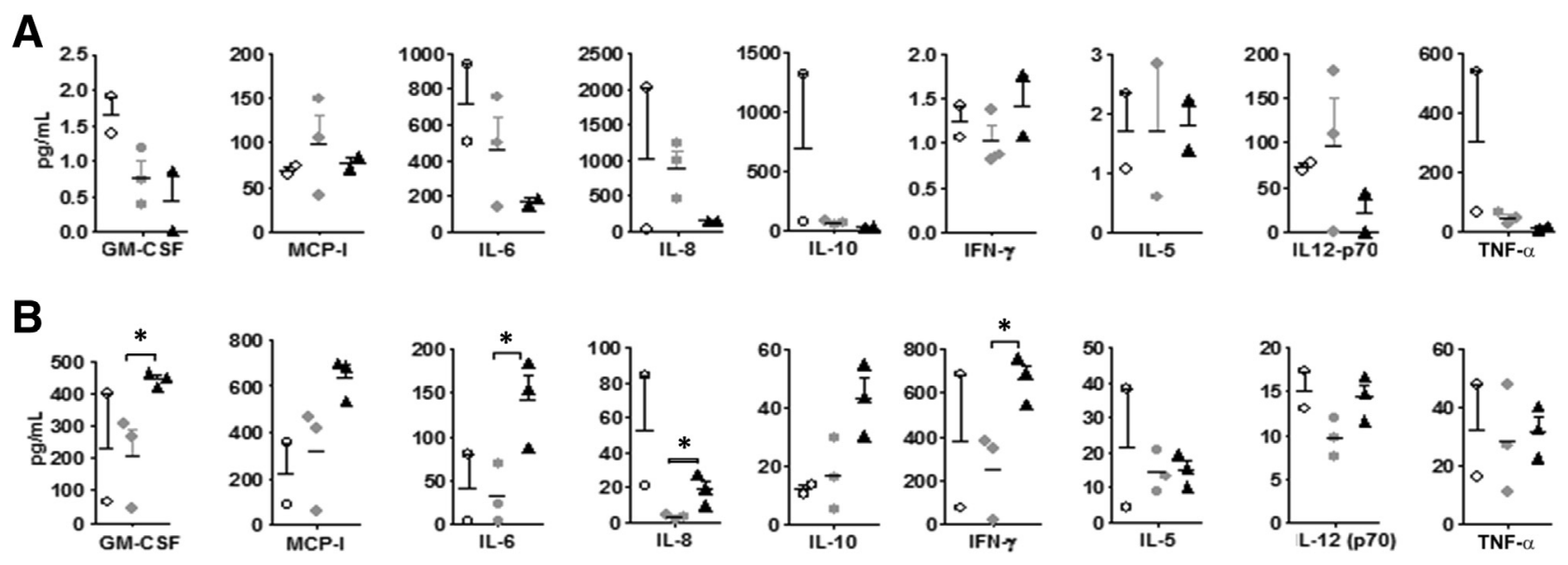

C
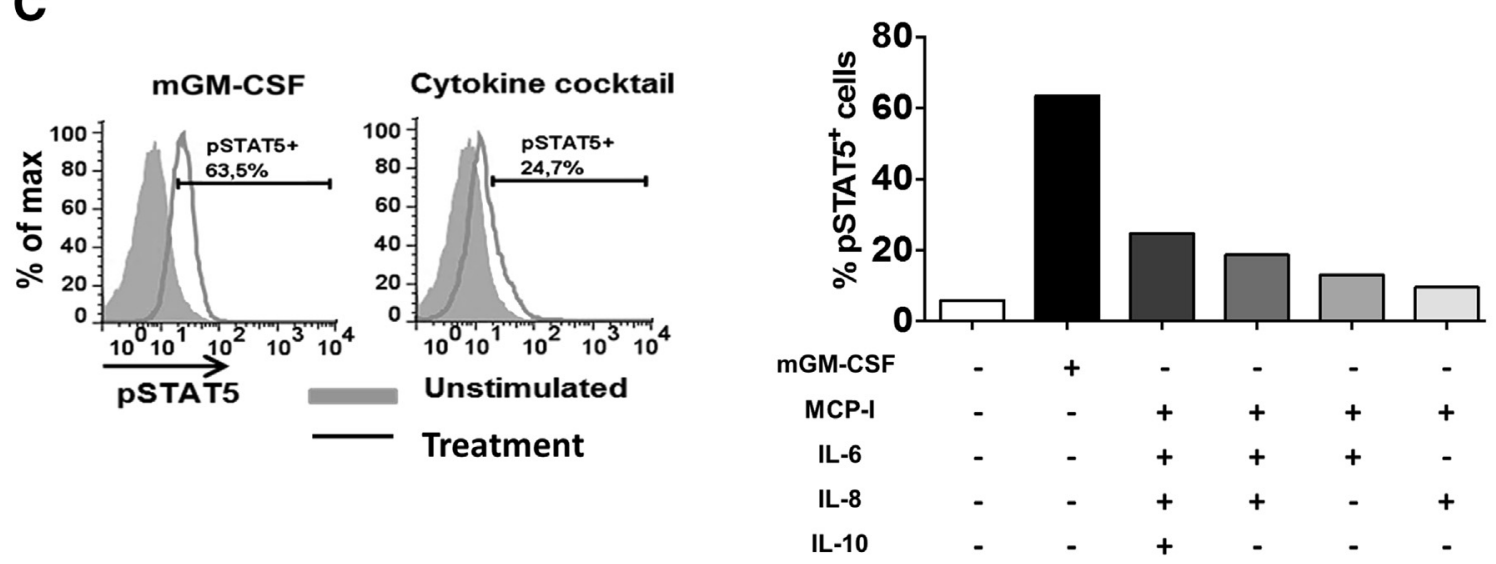

Figure 7 Analyses of human and mouse cytokines in plasma and correlation with the skin pathology. After euthanasia, murine and human Th1- (GM-CSF MCP-1, IL-6, IL-8, IL-10) and Th2-type cytokines [IFN- $\gamma$, IL-5, IL-12p70, tumor necrosis factor (TNF)- $\alpha$ ] were quantified in plasma by immune-bead array analyses. A: Mouse cytokines in plasma. B: Human cytokines in plasma. Concentration (pg/mL) of cytokines in plasma of control, iDCpp65-immunized, and iDCpp65/GVH-S mice, respectively. Statistical significance comparing iDCpp65-immunized mice without and with GVH-S is indicated on the graphs. C: Activation of the mouse macrophage cells (RAW264.7 cell line) with human cytokines. Cells were in vitro stimulated for 3 hours and pSTAT5 phosphorylation analyzed by flow cytometry. Bar graph indicates percentages of PSTAT5 ${ }^{+}$RAW264.7 cells at the end of the stimulation period. The table below the bar graph indicates the combination of cytokines used to stimulate the cells $(50 \mathrm{ng} / \mathrm{mL})$. As a positive control, activation with murine GM-CSF was used and nonstimulation was the negative control for the assay. Data are expressed as means \pm SEM (B). ${ }^{\star} P<0.05$. FACS, fluorescence-activated cell sorting; GM-CSF, granulocyte-macrophage colony-stimulating factor; MCP, monocyte chemoattractant protein.

GM-CSF were used as negative and positive controls, respectively. Three hours after the stimulation, the cells were immunostained for intracellular detection of phosphorylated STAT5 (pSTAT5). Cells stimulated with all human cytokines showed a 4.19-fold increase in the pSTAT5 expression compared to the nonstimulated cells. Even the human MCP-1 plus IL-8 combination showed an effect (1.62-fold compared to negative control) (Figure 7C). These in vitro results confirmed that high levels of human Th2-type cytokines, particularly in combinations, could cross the alleged species specificity and lead to the activation of mouse macrophages. Moreover, these results are important in view of new immunodeficient mouse strains engineered to produce several combinations of human cytokines. The combinations of several human cytokines in transgenic mice are thought to lead to a better engraftment and expansion of human cells, ${ }^{9}$ but on the other side, if these combinations are produced in excess, this can potentially result in inadvertent activation of mouse macrophages.

\section{Lymphocytes Isolated from LNs of iDCpp65/GVH-S Mice Show Superior pp65-Specific Immune Responses}

We next evaluated whether the skin pathology would be reflected in a xenograft outcome that could mask or fully abolish the pp65 antigenic specificity, because the immunization had been administered 6 months before onset of GVH-S. We used for these studies the same experimental conditions used by our group for short-term models of iDCpp65 efficacy testing at the level of pp65-specific T-cell responses. ${ }^{4,5}$ LNs from iDCpp65-immunized mice without $(n=3)$ or with GVH-S $(n=3)$ (Figure 8, A and B) were dissociated, and the single-cell suspensions were cryopreserved. Thawed pooled cells from each group were 


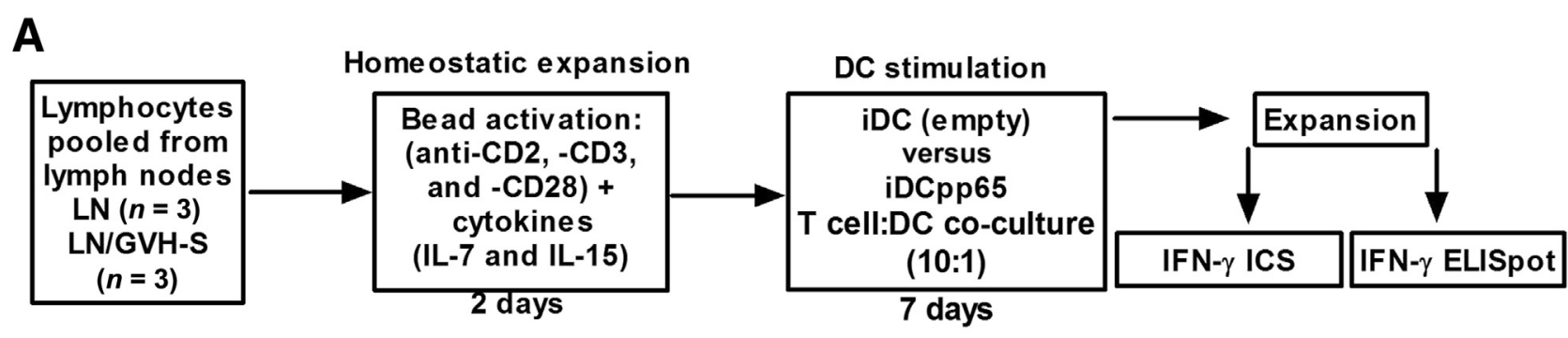

B

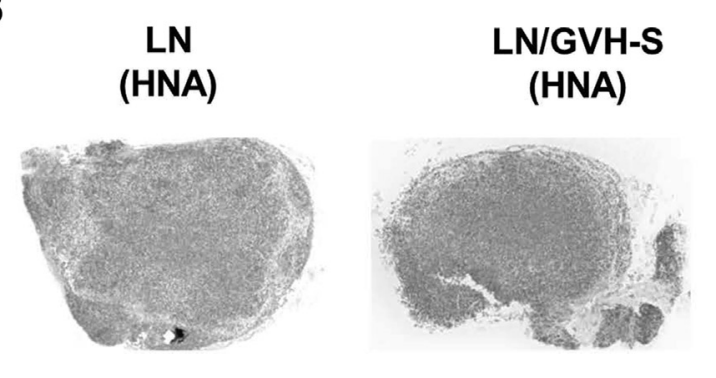

D

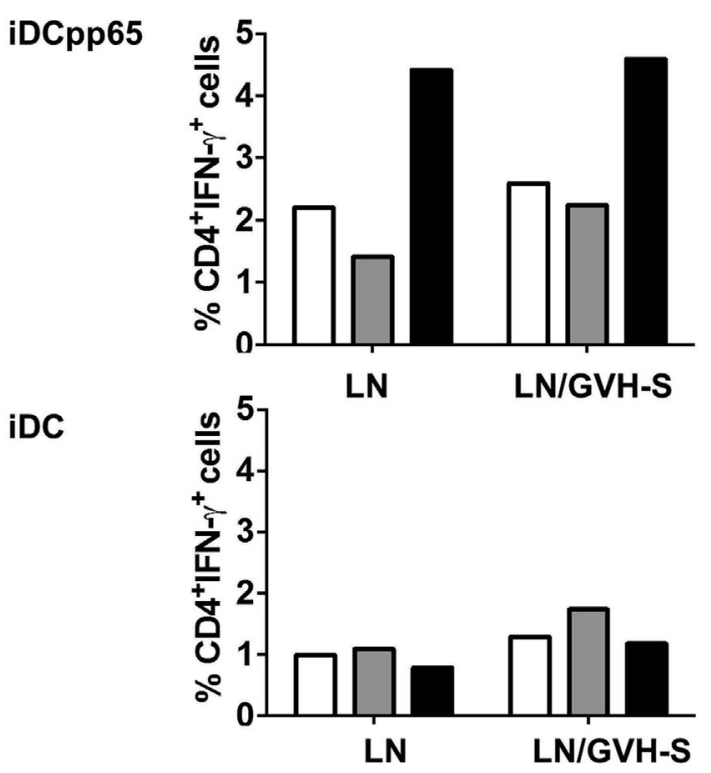

E

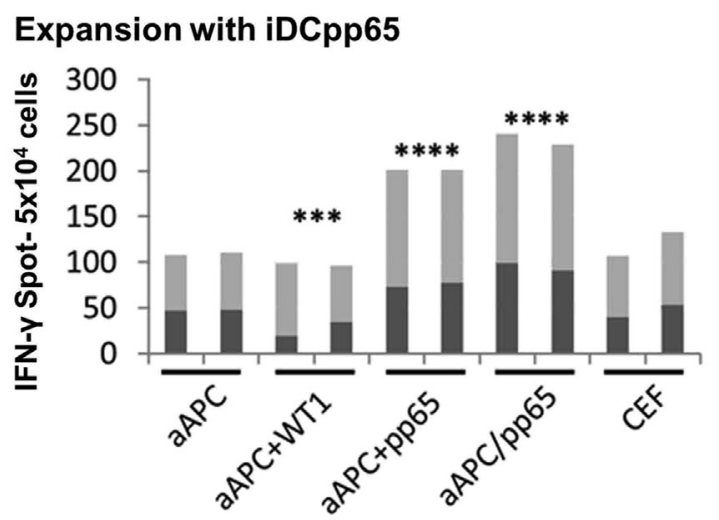

C

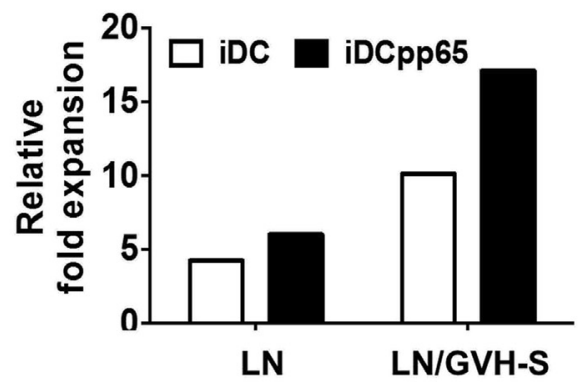

$\square$ No antigen

$\square$ WT1

pp65
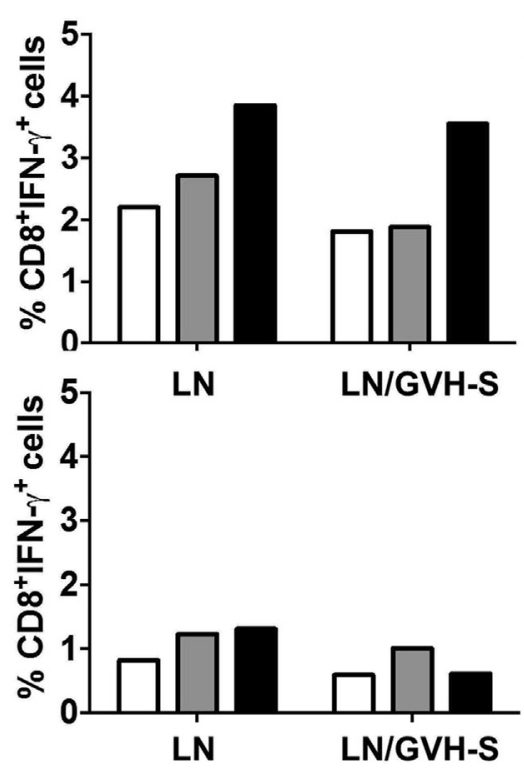

Expansion with iDC

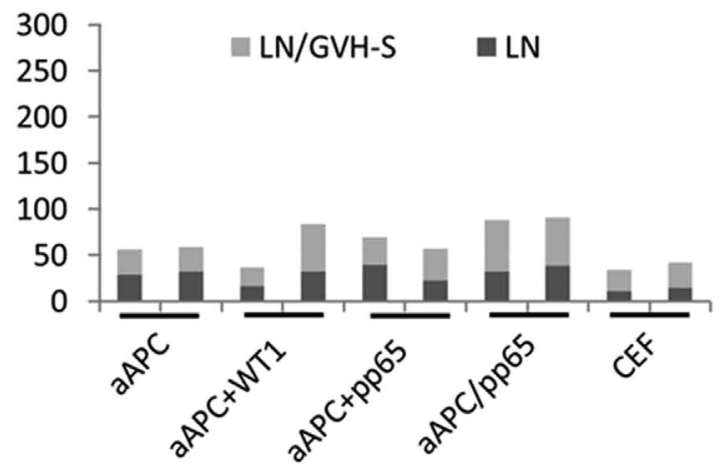


activated homeostatically with cytokines and activating beads for 48 hours and then co-cultured with autologous iDC (lacking antigens) or iDCpp65 at 1:10 DC/T-cell ratio for 7 days. Lymphocytes obtained from mice with GVH-S and restimulated with iDCpp65 showed the highest in vitro expansion (15-fold expansion, compared with fivefold expansion for mice with less pathology) (Figure 8C). T cells expanded with iDCpp65 were then restimulated with overlapping peptide pools corresponding to pp65, with an irrelevant (WT1) antigen pool, or were maintained without restimulation. Lymphocytes recovered from mice with and without GVH-S responded equally well to pp65 peptide stimulation. They produced IFN- $\gamma$, which was detected by intracellular staining of $\mathrm{CD}^{+}(\mathrm{LN}, 4.4 \%$; and LN/GVH-S, $4.59 \%$ ) and $\mathrm{CD} 8^{+} \mathrm{T}$ cells (LN, $3.85 \%$; and LN/GVH-S, 4.14\%). Restimulation with an irrelevant peptide pool (WT1) did not result in increased IFN- $\gamma$ production (Figure 8D). As a functional method to demonstrate that $\mathrm{T}$ cells derived from HLA-A*02.01 positive HSCs could recognize pp65 antigenic epitopes presented in the context of HLA-A*02.01 in aAPCs, we used an adaptation of our previously established IFN- $\gamma$ ELISPOT method. ${ }^{4}$ These aAPCs consisting of K562 cells stably expressing HLA$\mathrm{A} * 02.01$ were either loaded with a WT1 peptide pool $(\mathrm{aAPC}+\mathrm{WT1})$, with the pp65 peptide pool (aAPC + pp65), or transduced with LV for endogenous expression of full-length pp65 (aAPC/pp65). aAPCs not loaded with antigens were used as negative controls, and a CMV, EBV, and Flu peptide pool was used as a recall positive control. IFN- $\gamma$ ELISPOT analyses demonstrated that high frequencies of $\mathrm{T}$ cells expanded in vitro with iDCpp65 recognized the pp65 antigen presented by the aAPCs. Surprisingly, $\mathrm{T}$ cells expanded from LNs obtained from mice with GVH-S showed significantly higher frequencies of pp65-reactive $\mathrm{T}$ cells than mice without the pathology (Figure 8E and Supplemental Table S7). These results demonstrated that the reactivity against the pp65 antigen was sustained in iDCpp65-immunized mice even after $>23$ weeks after the first immunization. Furthermore, the occurrence of GVH-S did not have a negative impact on anti-pp65 responses, and it was positively correlated with the response to immunization. Taken together, the GVH-S pathology did not abolish a long-term antigen-specific T-cell memory response.

\section{Discussion}

We previously demonstrated the feasibility of production and quality control assessment of iDCpp65 under Good Manufacturing Practices-compliant conditions. ${ }^{7}$ Because the European Medicines Agency recommends that advanced therapeutic medicine products should be evaluated preclinically in relevant in vivo models, we performed pilot feasibility long-term studies to evaluate potency and safety of the iDCpp65 vaccine in Hu-HSC humanized mice. Consistent with our previous observations with shorter models (16 to 20 weeks after HSCT), the 36 weeks model confirmed the efficacy of iDCpp65 immunization: i) enhanced frequencies of $\mathrm{CD}^{+} \mathrm{T}$ cells in peripheral blood; ii) maturation of $\mathrm{T}$ cells toward effector memory $\mathrm{T}$ cells in spleen and LNs; and iii) long-term T-cell reactivity against the pp65 antigen. ${ }^{4,5}$

We did not observe weight loss, malignancies, or signs of systemic GVHD. Nevertheless, three mice immunized with iDCpp65 acquiring high levels of T-cell reconstitution and maturation at 30 weeks after HSCT eventually developed signs of a GVH-like effect on skin (which we called GVH-S), particularly on the head and flanks, and had to be sacrificed 3 weeks after. Post-mortem histopathological analyses of the affected skin showed prevalent infiltration of human $\mathrm{CD} 4^{+} \mathrm{T}$ cells surrounded by mouse macrophages in skin and spleen. Remarkably similar to the distribution of mouse $\mathrm{T}$ cells in skin of immunocompetent mice, ${ }^{10}$ we observed predominantly human $\mathrm{CD} 4^{+} \mathrm{T}$ cells over $\mathrm{CD} 8^{+} \mathrm{T}$ cells homing in the dermis. A great proportion of the human $\mathrm{CD} 4^{+} \mathrm{T}$ cells clustered on the hair follicles of the skin, and seemed to be in balance with the levels of $\mathrm{CD} 4^{+} \mathrm{T}$ cells in

Figure 8 Analyses of pp65-specific human immune responses measured from T cells isolated from lymph nodes and reactivated in vitro. A: Schematic representation of the experimental set-up to detect pp65 reactivity in T cells developed and expanded in humanized mice. Lymphocytes (corresponding to $>90 \%$ T cells) were pooled from lymph nodes of the iDCpp65-immunized mice and from the iDCpp65-immunized mice showing GVH-S. Lymphocytes were homeostatically activated with beads (anti-CD2, anti-CD3, and anti-CD28) plus human cytokines (IL-7 and IL-5) for 48 hours. Subsequently, lymphocytes were co-cultured and further expanded for 7 days with iDC (coexpressing GM-CSF/interferon (IFN)- $\alpha$ ) or with iDCpp65 (coexpressing GM-CSF/IFN- $\alpha /$ pp65) at 10:1 ratio. For IFN- $\gamma$ detection, intracellular staining followed by fluorescence-activated cell sorting (FACS) and ELISPOT assays were performed. B: Human cells in lymph nodes [anti-human nuclear antigen (HNA)]. Images of LN sections stained against anti-human nuclei (AHN) explanted from iDCpp65-immunized mice (LN) and iDCpp65/GVH-S (LN/GVH-S) showing an abundance of lymphocytes. C: T-cell expansion 7 days after the T/DC co-culture. Bar graph indicates the relative fold expansion of T cells further co-cultured with iDC or with iDCpp65. D: IFN- $\gamma$ intracellular staining. Detection of T-cell responses by intracellular staining of human IFN- $\gamma$ and FACS analyses. After expansion with the iDCs, T cells were incubated with a pp65 peptide pool or with a WT1 peptide pool. Controls were maintained with no antigenic peptides. Bar graphs show the frequencies of IFN- $\gamma^{+}$cells detected in the $\mathrm{CD}^{+}$and $\mathrm{CD}^{+} \mathrm{T}$-cell subpopulations. E: IFN- $\gamma$ ELISpot. Detection of T-cell responses by IFN- $\gamma$ ELISPOT using artificial antigen-presenting cells (aAPCs) not loaded with antigens (white bars), loaded with a WT1 peptide pool (punctuated bars), loaded with a pp65 peptide pool (gray bars), or expressing the full-length pp65 protein endogenously. A mix of highly antigenic peptides of CMV, EBV, and Flu (CEF) was used as a further control. Bar graphs indicate the counted number of detectable spots quantified for 5 $\times 10^{4}$ cells seeded on plates. Statistical difference between groups was analyzed by two-way analysis of variance with Bonferroni post $t$-test. Statistical significance comparing reactivity of T cells obtained with iDCpp65-immunized mice without and with GVH-S is indicated by symbols. $* * * P<0.001$, $* * * *<0.0001 \mathrm{LN}$ versus LN/GVH-S (two-way analysis of variance with Bonferroni post $t$-test). GM-CSF, granulocyte-macrophage colony-stimulating factor. 
circulation. In the perifollicular regions, clusters of $\mathrm{F} 4 / 80^{+}$ mouse macrophages were detected. Th2-type human cytokines (GM-CSF, MCP-1, IL-6, IL-8, and IL-10) were highly elevated in the plasma of mice with GVH-S. An experimental in vitro model showed that combinations of human MCP-1, IL-6, IL-8, and IL-10 were species cross-reactive and activated mouse macrophages. Although our previously published in vivo proof of concepts were reproducible and expanded to a long-term nonclinical model, the nature of the worrisome GVH-S pathology developing in humanized mice with the best immune reconstitution deserved clarification. ${ }^{4,5}$ As most studies using humanized mice are usually concluded within the first 20 weeks after HSCT (at the time when the human immune responses are rather weak), there is not much knowledge about the immune pathologies generated in humanized mice maintained longterm. Indeed, in the course of these studies, we started to observe similar skin pathology on the head and flanks of mice used to test stem cell units obtained from different umbilical cord blood donors and monitored for up to 25 weeks after HSCT. Of the 46 umbilical cord blood units tested, six produced a similar GVH-S in some mice, at different time points. We were able to retrieve the immune reconstitution data from three stem cell units to follow the frequencies of different T-cell subtypes for 25 weeks after transplantation. We split the mice between control $(n=7)$ and GVH-S mice $(n=3)$ (Supplemental Figure S2 and Supplemental Table S8). Although the numbers are too small for statistical comparisons, mice developing GVH-S showed a steeper reconstitution of $\mathrm{CD}^{+}, \mathrm{CD}^{+}, \mathrm{CD}^{+}$, and $\mathrm{EM} \mathrm{CD} 4^{+} \mathrm{T}$ cells. These data suggest that GVH-S depends on the stem cell donor and that the immunization with iDCpp65 may aggravate an underlying GVH-S, but does not cause it. Brehm and colleagues ${ }^{11}$ have reported that irradiated NOD/SCID/IL-2R $\gamma \mathrm{c}^{\text {null }}$ mice implanted with human fetal liver and thymus in the subrenal capsule and then injected i.v. with autologous fetal liver hematopoietic stem cells (bone marrow, liver, thymus, or BLT model), showed a reproducible development and maturation of human T cells. In this BLT model, human leukocyte levels increased in mice that had been engrafted for $>25$ weeks, and both $\mathrm{T}$ and $\mathrm{B}$ cells transitioned to activated phenotypes at these later time points. However, a fatal GVHD-like syndrome developed for approximately half of the mice at 28 weeks and all mice at 36 weeks after humanization. Activation of human $\mathrm{T}$ cells and increased levels of human Ig were seen. GVHD was not associated with the dose of CD34 ${ }^{+}$cells injected, with the initial levels of $\mathrm{T}$ cells observed at 12 weeks after humanization, with sex, with the loss of human regulatory $\mathrm{T}$ cells, or with the loss of mousederived intrathymic cellularity. Unfortunately, the interaction between human $\mathrm{T}$ lymphocytes and mouse macrophage cells was not reported for these studies. In another study with BLT-humanized mice using NOD-SCID and NOD/ SCID/IL-2R $\gamma \mathrm{c}^{\text {null }}$ strains, similar GVHD occurrences were reported. ${ }^{12}$ In this case, mice developed a spontaneous xenogenic GVHD characterized by lymphocytic infiltration and sclerosis of the skin, gastrointestinal tract, parotids, and lungs. Mice started dying to disease at 15 weeks after reconstitution; by 36 weeks, $75 \%$ of the mice had died. Histologically, skin involvement was characterized by infiltration of the epidermis and hair follicles with human lymphocytes. Colitis was characterized by a lamina propria infiltrate. Because specific donor HLA class I alleles have been associated with the incidence and manifestations of human GVHD, the effects of HLA class I from 50 donor cohorts were typed for HLA class I alleles. This genetic analysis indicated that particular donor HLA class I alleles influenced the risk for the development of xenogeneic GVHD in the BLT mice. At a cellular level, this xenogeneic GVHD was associated with the infiltration of human IFN$\gamma$-producing human $\mathrm{CD}^{+} \mathrm{T}$ cells into the skin. Nevertheless, human Th2-type cytokines (IL-4, IL-10) did not account for the pathology. Interestingly, they reported that GVHD induced a mixed M1/M2 polarization phenotype in a dermal murine $\mathrm{CD} 11 \mathrm{~b}^{+}$, major histocompatibility complex class $\mathrm{II}^{+}$macrophage population. This macrophage population entirely of mouse origin expressed Cox2, Nos2, Arg1, and $Y m 1$, IL-25, and IL-13R $\alpha 1$, which were previously shown by the same group ${ }^{12}$ to be associated with an IL13-driven expression signature in murine GVHD and human scleroderma, respectively. Although a strong association between the HLA type, the presence of human $\mathrm{CD}^{+}{ }^{+} \mathrm{T}$ cells, and mouse macrophages in the skin suggested their involvement in disease, a working model for development of GVHD was not specified. More important, independent results of these two groups using the BLT model relating the participation of human activated $\mathrm{CD} 4^{+} \mathrm{T}$ cells in skin inflammation of humanized mice seemed similar to our data. In our model, however, the lethality was much lower in occurrence (three of eight mice in one of the HSCT reconstitutions) and milder in distribution (only affecting the skin, but not colon or lungs). In addition, we ratified the involvement of activated mouse macrophages and demonstrated that human Th2-type cytokines abundant in the plasma of the affected mice, particularly in combinations, could directly activate mouse macrophages. MCP-1 plays a central role in the recruitment of monocytes/macrophages associated with several inflammatory diseases and malignancies. MCP-1 was previously shown to activate the mouse macrophage model cell line RAW264.7, making them cytotoxic to mouse aortic smooth muscle cells through a FasL-Fas-Caspase8-RIP1-mediated mechanism. ${ }^{13}$ Interestingly, in our study, murine and human MCP-1 were both detectable in the plasma of all mice. Our data might infer a cumulative effect of human and mouse MCP-1 acting on mouse macrophages, which, in turn, exert their cytotoxic effects on the mouse keratinocytes. Human IL-6 is also another crucial cytokine, considered a biomarker for prediction of CRS. ${ }^{8}$ IL-6 is a proinflammatory cytokine involved in several processes of the immune system, B-cell differentiation, autoantibody production, and neutrophil 
trafficking. ${ }^{14}$ In a SCID-beige mouse model, the IL-6-mediated toxicities could be ameliorated by depletion of macrophages. ${ }^{15}$ Our data showed that mice showing a preponderance of $\mathrm{CD}^{+}$mature $\mathrm{T}$ cells and high levels of human IL-6 presented with GVH-S. Incidentally, human IL-6 was able to activate the mouse RAW264.7 cell line, indicating a potential role of human IL-6 in GVH-S development. More important, CRS has been commonly observed in lymphoma and leukemia patients treated with autologous $\mathrm{T}$ cells engineered for expression of chimeric antigen receptors directed against CD19 (CART 19). These CRS patients showed significant elevation of IL-6, IL-10, and IFN- $\gamma \cdot{ }^{16,17}$ IL-6 blockade (with tocilizumab) has been shown to ameliorate the effects of CRS without affecting the T-cell efficacy. ${ }^{18,19}$ This cytokine elevation pattern is similar to macrophage activation syndrome (MAS). Patients after treatment with CART 19 who developed severe CRS showed a clinical pathology also resembling MAS. Thus, the pathophysiology of the two diseases is strikingly similar and may be interconnected. The patient's genetic predisposition seems to be another factor in the progression from CRS to MAS, such as polymorphisms seen in the perforin gene. ${ }^{19}$ Currently, inhibitors of both MCP-1 and IL-6 are being considered in managing the CRS after CART-cell immunotherapies. ${ }^{14}$ Taken together, this evidence pointed out that the high activation of xenogeneic and/or antigenspecific human T cells resulted in a CRS-like effect, leading ultimately to murine MAS and skin tissue destruction. Although the situation in these humanized mice may be even more complex than in humans (because of the complete and still unpredictable effects of the mouse-human major histocompatibility complex mismatch), it would be interesting to use these models to predict preclinically if some CART designs are more prone than others to cause MAS, and maybe how to remediate it with monoclonal antibodies blocking specific cytokines. Some of the advantages of the Hu-HSC model over BLT mice have been postulated by Zack and colleagues, ${ }^{20}$ as follows: i) easier to prepare and lower cost, ii) negligible GVHD and longer life span, and iii) safety and toxicity assessed for several weeks. But as Hu-HSC models continuously mature and improve with new mouse strains and adoptive transfer of cells and/or tissues, the next frontier is the demand for long-term studies: are these indeed feasible, reproducible, and predictive for nonclinical good laboratory practice studies? The main identified limitations of current humanized mouse model are as follows ${ }^{20}:$ i) human leukocyte antigen (HLA) molecules are required for appropriate T-cell selection after human HSC engraftment; ii) many human cytokines and other factors are species specific; iii) remaining innate immunity impairs engraftment; iv) impaired humoral immune responses, low level of immunoglobulin production, and impaired immunoglobulin class switching; v) impaired lymph node development and poorly developed germinal centers. In this current work, we confirmed that iDCpp65 applied after Hu-HSCT effectively boosted the human
T-cell reconstitution counteracting some of these limiting issues in humanized mice. Nevertheless, the unexpected long-term consequence of harnessing human Th2-type $\mathrm{CD}^{+} \mathrm{T}$ cells in humanized mice was the cross-activation of pathological mouse macrophages. Therefore, the ultimate demonstration of clinical potency and safety for iDCpp65 remains to be performed in humans, with the foreseen caveat that CRS or MAS could also occur as adverse effects of enhanced $\mathrm{CD} 4^{+} \mathrm{T}$-cell responses.

\section{Acknowledgments}

We thank all current and past members of the Regenerative Immune Therapies Applied laboratory for their valuable contributions. We also thank the consortium working on a clinical translation of iDCpp65 to protect high-risk patients against human cytomegalovirus (Profs. Heiko von der Leyen, Ulrike Köhl, and Michael Schmitt, and Dr. Ulrich Grigoleit).

B.S.S. designed and conducted experiments, analyzed data, and wrote the first draft of the manuscript; V.V, S.J.T., and A.S. assisted in the execution of Hu-HSC mice maintenance, monitoring, euthanasia, and sample preparation for the experiments and pathology; V.V. revised the humanized mouse database, provided data for GVH-S in nonimmunized mice, and performed statistical analyses and revised figures and tables; L.G. prepared and analyzed stem cells for transplantation; S.R., D.S., K.S., and A.B. performed immunohistopathological analyses, prepared the slides, and interpreted the data; V.N. performed the mouse cytokine array analyses; C.F. performed the human cytokine array analyses; A.M and T.M. assisted in the planning of evaluation of RAW264.7 cytokine stimulation by intracellular detection of pSTAT5; C.v.K. assisted in the procurement and collection of HSC for the studies; H.W. participated in the discussions with the regulatory agency in Germany [Paul-Ehrlich-Institut (PEI)] for the conception of the pharm-tox studies; L.M.S. performed and revised statistical analyses; A.G. participated with clinical input for the project and edited the final revised manuscript; R.S. planned the project, obtained funding and regulatory approvals, designed experiments, enrolled collaborators, interpreted the data, and edited the final manuscript.

\section{Supplemental Data}

Supplemental material for this article can be found at http://dx.doi.org/10.1016/j.ajpath.2017.02.015.

\section{References}

1. Shultz LD, Ishikawa F, Greiner DL: Humanized mice in translational biomedical research. Nat Rev Immunol 2007, 7:118-130

2. Shultz LD, Brehm MA, Garcia-Martinez JV, Greiner DL: Humanized mice for immune system investigation: progress, promise and challenges. Nat Rev Immunol 2012, 12:786-798 
3. Yamauchi T, Takenaka K, Urata S, Shima T, Kikushige Y, Tokuyama T, Iwamoto C, Nishihara M, Iwasaki H, Miyamoto T, Honma N, Nakao M, Matozaki T, Akashi K: Polymorphic Sirpa is the genetic determinant for NOD-based mouse lines to achieve efficient human cell engraftment. Blood 2013, 121:1316-1325

4. Daenthanasanmak A, Salguero G, Sundarasetty BS, Waskow C, Cosgun KN, Guzman CA, Riese P, Gerasch L, Schneider A, Ingendoh A, Messerle M, Gabaev I, Woelk B, Ruggiero E, Schmidt M, von Kalle C, Figueiredo C, Eiz-Vesper B, von Kaisenberg C, Ganser A, Stripecke R: Engineered dendritic cells from cord blood and adult blood accelerate effector $\mathrm{T}$ cell immune reconstitution against HCMV. Mol Ther Methods Clin Dev 2015, 1:14060

5. Salguero G, Daenthanasanmak A, Munz C, Raykova A, Guzman CA, Riese P, Figueiredo C, Langer F, Schneider A, Macke L, Sundarasetty BS, Witte T, Ganser A, Stripecke R: Dendritic cell-mediated immune humanization of mice: implications for allogeneic and xenogeneic stem cell transplantation. J Immunol 2014, 192:4636-4647

6. Daenthanasanmak A, Salguero G, Borchers S, Figueiredo C, Jacobs R, Sundarasetty BS, Schneider A, Schambach A, Eiz-Vesper B, Blasczyk R, Weissinger EM, Ganser A, Stripecke R: Integrasedefective lentiviral vectors encoding cytokines induce differentiation of human dendritic cells and stimulate multivalent immune responses in vitro and in vivo. Vaccine 2012, 30:5118-5131

7. Sundarasetty BS, Kloess S, Oberschmidt O, Naundorf S, Kuehlcke K, Daenthanasanmak A, Gerasch L, Figueiredo C, Blasczyk R, Ruggiero E, Fronza R, Schmidt M, von Kalle C, Rothe M, Ganser A, Koehl U, Stripecke R: Generation of lentivirus-induced dendritic cells under GMP-compliant conditions for adaptive immune reconstitution against cytomegalovirus after stem cell transplantation. J Transl Med 2015, 13:240

8. Lee DW, Gardner R, Porter DL, Louis CU, Ahmed N, Jensen M, Grupp SA, Mackall CL: Current concepts in the diagnosis and management of cytokine release syndrome. Blood 2014, 124:188-195

9. Rongvaux A, Willinger T, Martinek J, Strowig T, Gearty SV, Teichmann LL, Saito Y, Marches F, Halene S, Palucka AK, Manz MG, Flavell RA: Development and function of human innate immune cells in a humanized mouse model. Nat Biotechnol 2014, 32:364-372

10. Collins N, Jiang X, Zaid A, Macleod BL, Li J, Park CO, Haque A, Bedoui S, Heath WR, Mueller SN, Kupper TS, Gebhardt T, Carbone FR: Skin CD4(+) memory T cells exhibit combined cluster-mediated retention and equilibration with the circulation. Nat Commun 2016, 7:11514

11. Covassin L, Jangalwe S, Jouvet N, Laning J, Burzenski L, Shultz LD, Brehm MA: Human immune system development and survival of nonobese diabetic (NOD)-scid IL2rgamma(null) (NSG) mice engrafted with human thymus and autologous haematopoietic stem cells. Clin Exp Immunol 2013, 174:372-388

12. Greenblatt MB, Vrbanac V, Tivey $T$, Tsang $K$, Tager AM, Aliprantis AO: Graft versus host disease in the bone marrow, liver and thymus humanized mouse model. PLoS One 2012, 7:e44664

13. Wang Q, Ren J, Morgan S, Liu Z, Dou C, Liu B: Monocyte chemoattractant protein-1 (MCP-1) regulates macrophage cytotoxicity in abdominal aortic aneurysm. PLoS One 2014, 9:e92053

14. Maude SL, Barrett D, Teachey DT, Grupp SA: Managing cytokine release syndrome associated with novel $\mathrm{T}$ cell-engaging therapies. Cancer J 2014, 20:119-122

15. van der Stegen SJ, Davies DM, Wilkie S, Foster J, Sosabowski JK, Burnet J, Whilding LM, Petrovic RM, Ghaem-Maghami S, Mather S, Jeannon JP, Parente-Pereira AC, Maher J: Preclinical in vivo modeling of cytokine release syndrome induced by ErbB-retargeted human $\mathrm{T}$ cells: identifying a window of therapeutic opportunity? J Immunol 2013, 191:4589-4598

16. Porter DL, Levine BL, Kalos M, Bagg A, June CH: Chimeric antigen receptor-modified $\mathrm{T}$ cells in chronic lymphoid leukemia. N Engl J Med 2011, 365:725-733

17. Klinger M, Brandl C, Zugmaier G, Hijazi Y, Bargou RC, Topp MS, Gokbuget N, Neumann S, Goebeler M, Viardot A, Stelljes M, Bruggemann M, Hoelzer D, Degenhard E, Nagorsen D, Baeuerle PA, Wolf A, Kufer P: Immunopharmacologic response of patients with Blineage acute lymphoblastic leukemia to continuous infusion of T cellengaging CD19/CD3-bispecific BiTE antibody blinatumomab. Blood 2012, 119:6226-6233

18. Teachey DT, Rheingold SR, Maude SL, Zugmaier G, Barrett DM, Seif AE, Nichols KE, Suppa EK, Kalos M, Berg RA, Fitzgerald JC, Aplenc R, Gore L, Grupp SA: Cytokine release syndrome after blinatumomab treatment related to abnormal macrophage activation and ameliorated with cytokine-directed therapy. Blood 2013, 121: 5154-5157

19. Grupp SA, Kalos M, Barrett D, Aplenc R, Porter DL, Rheingold SR, Teachey DT, Chew A, Hauck B, Wright JF, Milone MC, Levine BL, June $\mathrm{CH}$ : Chimeric antigen receptor-modified $\mathrm{T}$ cells for acute lymphoid leukemia. N Engl J Med 2013, 368:1509-1518

20. Akkina R, Allam A, Balazs AB, Blankson JN, Burnett JC, Casares S, Garcia JV, Hasenkrug KJ, Kashanchi F, Kitchen SG, Klein F, Kumar P, Luster AD, Poluektova LY, Rao M, SandersBeer BE, Shultz LD, Zack JA: Improvements and limitations of humanized mouse models for HIV research: NIH/NIAID "Meet the Experts" 2015 Workshop Summary. AIDS Res Hum Retroviruses 2016, 32:109-119 